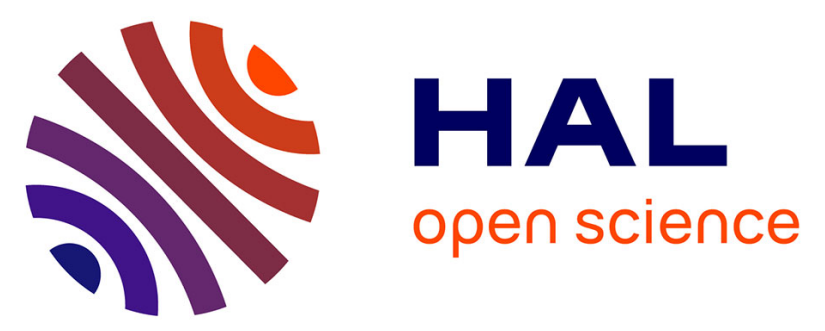

\title{
Impact of concurrent training on body composition and gut microbiota in postmenopausal women with overweight or obesity
}

\author{
Marine Dupuit, Mélanie Rance, Claire Morel, Patrice Bouillon, Audrey
}

Boscaro, Nathalie Boisseau, Martin Vincent, Emilie Vazeille, Nicolas Barnich, Benoit Chassaing

\section{To cite this version:}

Marine Dupuit, Mélanie Rance, Claire Morel, Patrice Bouillon, Audrey Boscaro, et al.. Impact of concurrent training on body composition and gut microbiota in postmenopausal women with overweight or obesity. Medicine and Science in Sports and Exercise, 2022, 54 (3), pp.517-529. 10.1249/MSS.0000000000002809 . hal-03384031

\section{HAL Id: hal-03384031 \\ https://hal.inrae.fr/hal-03384031}

Submitted on 25 Nov 2021

HAL is a multi-disciplinary open access archive for the deposit and dissemination of scientific research documents, whether they are published or not. The documents may come from teaching and research institutions in France or abroad, or from public or private research centers.
L'archive ouverte pluridisciplinaire HAL, est destinée au dépôt et à la diffusion de documents scientifiques de niveau recherche, publiés ou non, émanant des établissements d'enseignement et de recherche français ou étrangers, des laboratoires publics ou privés.

\section{(ㅇ)(1) $\$$}

Distributed under a Creative Commons Attribution - NonCommercial - NoDerivatives $\mid 4.0$ 


\section{Impact of concurrent training on body composition and gut microbiota in}

\section{2 postmenopausal women with overweight or obesity}

3 Marine Dupuit ${ }^{1}$, Mélanie Rance ${ }^{2}$, ${\text { Claire } \text { Morel }^{2} \text {, Patrice Bouillon }}^{3}$, Audrey Boscaro ${ }^{1}$, Vincent

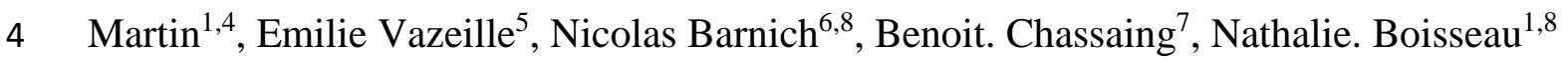

$6{ }^{1}$ : Laboratory of the Metabolic Adaptations to Exercise under Physiological and Pathological

7 Conditions (AME2P), Clermont Auvergne University, UR 3533, 63171 Clermont-Ferrand,

8 France

9 marine.dupuit@uca.fr; nathalie.boisseau@uca.fr; vincent.martin@uca.fr;

10 audrey.boscaro@etu.uca.fr

11 2: Center of Resources, Expertise and Performance in Sports (CREPS), 03321 Bellerive-sur12 Allier, France.

melanie.rance@creps-vichy.sports.gouv.fr; claire.morel@creps-vichy.sports.gouv.fr

$143^{3}$ : Department of Cardiology, Vichy Hospital, 03300 Vichy, France.

15 patrice.bouillon@ch-vichy.fr

16 4: University Institute of France (IUF), 75000 Paris, France.

17 vincent.martin@uca.fr

5: Department of Cardiology, University Hospital of Clermont-Ferrand, 63000 Clermont-

19 Ferrand, France.

evazeille@chu-clermontferrand.fr

$21{ }^{6}$ : Microbes, Intestine, Inflammation and Susceptibility of the Host (M2iSH), UMR 1071, USC

INRAE 2018, Université Clermont Auvergne, 63000 Clermont-Ferrand, France.

nicolas.barnich@uca.fr

7: Inserm U1016, Team 'Mucosal microbiota in chronic inflammatory diseases', CNRS UMR 
benoit.chassaing@inserm.fr

27

8: Human Nutrition Research Center of Auvergne - Rhône-Alpes (CNRH-AURA), 63000 Clermont-Ferrand, France

nathalie.boisseau@uca.fr

\section{Corresponding author}

Professor Nathalie Boisseau (PhD). Laboratoire des Adaptations Métaboliques à l'Exercice en conditions Physiologiques et Pathologiques (AME2P), 3 rue de la Chebarde, 63178, Aubière Cedex, France.

Phone number: 00334734055 19; e-mail: nathalie.boisseau@uca.fr

(1)

\section{ABSTRACT}

Purpose: Menopause tends to be associated with an increased risk of obesity and abdominal fat mass (FM) and is associated with lower intestinal species diversity. The aim of this study was to determine the effects of a high-intensity interval training and resistance training (HIIT $+\mathrm{RT}$ ) program on body composition and intestinal microbiota composition in overweight or obese postmenopausal women.

Methods: Participants $(n=17)$ were randomized in two groups: HIIT + RT group $(3 \times /$ week, 12 weeks) and control group without any training. Dual-energy X-ray absorptiometry was used to measure whole-body and abdominal/visceral FM and fat-free mass. Intestinal microbiota composition was determined by $16 \mathrm{~S}$ rRNA gene sequencing at baseline and at the study end, and the diet controlled.

Results: Compared with sedentary controls, physical fitness (Maximal Oxygen Consumption, Peak Power Output) increased, total abdominal and visceral FM decreased, and segmental 
muscle mass increased in the training group. Although the HIIT + RT protocol did not modify $\alpha$-diversity and taxonomy, it significantly influenced microbiota composition. Moreover, various intestinal microbiota members were correlated with HIIT + RT-induced body composition changes, and baseline microbiota composition predicted the response to the HIIT + RT program.

Conclusions: HIIT + RT is an effective modality to reduce abdominal/visceral FM and improve physical capacity in non-dieting overweight or obese postmenopausal women. Training modified intestinal microbiota composition and the response to training seems to depend on the initial microbiota profile. More studies are needed to determine whether microbiota composition could predict the individual training response.

Key words: menopause, concurrent training, fat mass, abdominal/visceral fat mass, gut microbiota composition.

\section{INTRODUCTION}

Obesity has reached epidemic proportions and is a major contributor to the global burden of chronic disease and disability. Obesity is a complex multifactorial disease mainly favored by sedentary lifestyle, low physical activity level, and high consumption of processed and highcalorie foods (1). Fat mass (FM) accumulation and metabolic disturbances are associated with greater risk of cardiovascular diseases (CVD). It is recognized that abdominal and particularly visceral adiposity increase the risk of developing obesity-related complications compared with subcutaneous adiposity (2). The reduction of visceral adipose tissue is naturally associated with a decreased risk of metabolic syndrome and CVD (3). 
The prevalence of obesity is higher in post- than in pre-menopausal women (4). Menopause is associated with energy metabolism changes, particularly decreased fat oxidation at rest and during exercise (5), lower spontaneous physical activity (6) and consequently, lower total energy expenditure (5). Estrogen production deficiency also favors muscle mass loss and FM gain with higher central body fat distribution (i.e. abdominal/visceral deposits) and consequently higher CVD risk $(7,8)$.

The combination of endurance (aerobic) and resistance (strength) exercise modalities can contribute to preventing and treating obesity-associated abnormalities (9). Recent studies by our laboratory showed that 3-4-month programs of high-intensity interval training (HIIT) (10) or HIIT plus resistance training (RT) (11) are safe and efficient strategies to significantly reduce total and (intra-)abdominal FM in women with overweight or obesity. Compared with moderate intensity continuous training, the HIIT + RT combination also increases muscle mass in this population (11), leading to higher resting metabolic rate and 24-h energy expenditure. Recently, Rashiti et al. confirmed that compared with moderate intensity continuous training + RT, HIIT + RT has a greater effect on obesity-related parameters in postmenopausal women (12).

Gut microbial dysbiosis/unfavorable composition might also promote obesity development. It is acknowledged that gut microorganisms are important pathogenic factors, contributing to systemic inflammation, insulin-resistance and directly or indirectly, to body composition changes $(13,14)$. Similarly, obesity has been associated with significant changes in gut microbiota composition and metabolic functions (including a decrease of $\alpha$ and $\beta$ diversity and/or an imbalance between benefic and pathogenic bacteria that could alter the carbohydrate metabolism), indicating a two-way relationship between intestinal microbiota and human metabolism (14). The gut microbiota is mainly shaped by diet (13), but regular physical activity is also emerging as an important modulator of gut microbial community structure and diversity 
(15-17). Higher levels of physical activity (total volume and intensity) are associated with higher fecal bacterial diversity and with the increased representation of some phyla and certain short-chain fatty acids in the feces of healthy adults $(18,19)$. Menopause and more generally aging reduce intestinal species diversity, an effect that might contribute to the high prevalence of obesity in this population (20). Few studies in humans have directly examined the effect of regular physical activity on gut microbiota (20-24) and none used the HIIT + RT combination. Therefore, the main objective of this study was to evaluate the impact of a 12-week HIIT + RT program on body composition and gut microbial community structure and diversity in nondieting postmenopausal women with overweight or obesity. We hypothesized that the concurrent training HIIT + RT would decrease total and (intra-)abdominal adipose tissue and that these alterations would be associated with a favorable alteration of the gut microbiota composition.

\section{METHODS}

On the basis of previous results on visceral FM loss after a 3-month HIIT + RT program in postmenopausal women (11), sample size was estimated before the study beginning to ensure a statistical power of $80 \%$. Considering a two-sided type I error at $5 \%$, a minimal difference of $1.5 \mathrm{~kg}$ in visceral FM loss (standard deviation $=1.0$ ) could be detected with $n=7$ participants per group. The sample size was increased to $n=14$ and 15 per group to take into account participants lost to follow-up.

This study was approved by the relevant ethics committee (Comité de Protection des Personnes Ouest VI, CPP 1141 HPS1) and was registered on ClinicalTrials.gov (ClinicalTrials.Gov: NCT03940924). Participants were recruited via flyers, posters and adverts on websites and social networks. Before the study, participants were given explanations on the study aims and methods and their written informed consent was collected. 


\section{Participants}

130 For practical and feasibility reasons the protocol was carried out in four waves (February to May and September to December 2019, February to May and September to December 2020), and the third wave was stopped due to the COVID-19 pandemic.

Thirty-three participants were recruited according to the following inclusion criteria: postmenopausal women, body mass index $(\mathrm{BMI})>25 \mathrm{~kg} \cdot \mathrm{m}^{-2}$ and $\leq 40 \mathrm{~kg} \cdot \mathrm{m}^{-2}$, and stable eating habits and physical activity for at least 3 months. Non-inclusion criteria were: medical contraindications to intense physical activity, painful joints, taking hormone replacement therapy and taking antibiotics for at least 3 months. Finally, 29 women were selected for the 12-week study (Figure 1). None of them had history of chronic arterial or respiratory diseases, CVD, or endocrine disorders. All participants reported low levels of physical activity, based on the Global Physical Activity Questionnaire (GPAQ) result (25). Participants were subdivided in two groups in order of inclusion: intervention group (HIIT + RT, $n=14$ ) and control group without training program $(\mathrm{CONT}, n=15)$.

\section{EXPERIMENTAL DESIGN}

Anthropometric and body composition measurements. Body weight was measured to the nearest $0.1 \mathrm{~kg}$ on a Seca 709 scale (Balance Seca 709, France), with participants wearing only underwear. Height was measured to the nearest $0.5 \mathrm{~cm}$ using a wall-mounted stadiometer. Body mass index (BMI, kg.m ${ }^{-2}$ ) was calculated as body mass $(\mathrm{kg})$ divided by the square of height 
$\left(\mathrm{m}^{2}\right)$. Waist circumference (WC, $\mathrm{cm}$ ) was measured midway between the last rib and upper iliac crest, and hip circumference at the level of the femoral trochanters. Both measures were taken in standing position with a measuring tape. The sagittal abdominal diameter (supine abdominal height) was measured with a Holtain-Kahn abdominal caliper (Holtain Limited, Crymych, Pembs, UK) to the nearest $\mathrm{mm}$ in the sagittal plane at the level of the iliac crests (L4-L5) during normal expiration, with the subject lying supine on a firm bench with knees bent. Abdominal skinfold thickness was measured at four different sites (at $12 \mathrm{~cm}$ and $7 \mathrm{~cm}$ to the right and left of the navel) with a Harpenden Skinfold Caliper (Mediflex Corp., Long Island, NY, USA), and the mean subcutaneous abdominal skinfold thickness was then calculated. The study end. skinfold thickness and abdominal height, as previously described (11).

Fat and muscle mass localization. Total body and regional fat mass (FM) and free fat mass (FFM) (expressed as $\mathrm{kg}$ and \% of body mass) were measured with a dual-energy X-ray absorptiometry scanner (QDR-4500A, Hologic, Inc., Marlborough, MA, USA). Muscle mass was calculated by removing the bone mineral content from the FFM. Participants in underwear were placed in the middle of the table in supine position. Whole body scanning was performed for $3 \mathrm{~min}$ and the same operator analyzed all collected data. Two regions of interest were manually isolated by the same investigator: the area from L2-L3 to the pubic rami to determine the total abdominal FM $(\mathrm{kg})$, and the area delineated by the upper border formed by an oblique line passing through the femoral neck to the horizontal line passing through the knee to determine the thigh muscle mass $(\mathrm{kg})(26)$. Total visceral FM $(\mathrm{kg})$ was estimated from the total abdominal FM obtained by dual-energy X-ray absorptiometry, mean subcutaneous abdominal skinfold thickness and abdominal height, as previously described (11). 
Preliminary visit - maximal exercise testing. $\mathrm{VO}_{2 \max }$ was measured during a graded exhaustive exercise test on a cycle ergometer (Ergoline, Bitz, Germany). After a 3-min warmup at $30 \mathrm{~W}$, power output was increased by 10 Watts per minute until the participant's exhaustion (the test lasted between 10 and 15 minutes after warm-up). Participants were strongly encouraged by the experimenters throughout the test to perform a maximal effort. Respiratory gases $\left(\mathrm{VO}_{2}\right.$ and $\left.\mathrm{VCO}_{2}\right)$ were measured breath-by-breath through a mask connected to $\mathrm{O}_{2}$ and $\mathrm{CO}_{2}$ analyzers (MasterScreen ${ }^{\mathrm{TM}} \mathrm{CPX}$, Care Fusion, Le Pont-de-Claix, France). $\mathrm{VO}_{2 \max }$ was determined as the highest oxygen uptake during a $15 \mathrm{~s}$ period. Ventilatory parameters were averaged every 30s. Heart activity was monitored by ECG throughout the test, and heart rate recorded continuously. $\mathrm{VO}_{2 \max }$ achievement was based on the following criteria: 1) oxygen uptake reaching a plateau with increasing work rate;2) respiratory exchange ratio values higher than 1.1 ; and 3$)$ maximal heart rate $\left(\mathrm{HR}_{\max }\right)$ within $10 \%$ of the age-predicted maximal values. The Peak Power Output (PPO), expressed in watts or watts.kg-1, was considered the highest power measured at $\mathrm{VO}_{2 \max }$.

(1)

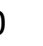

1

\section{Microbiota composition analysis by Illumina sequencing}

Participants received a plastic tube to collect their stool, within $24 \mathrm{~h}$ before the study initiation (first exercise session for the training group) and at the study end (24h after last exercise session for the training group), and were instructed to store the stool sample in a plastic bag in their home freezer. Upon receipt, samples were stabilized in RNA Later (Sigma Aldrich, MI, USA) and stored at $-80^{\circ} \mathrm{C}$ until processing.

Genomic DNA from fecal samples was extracted using the Maxwell ${ }^{\circledR}$ RSC PureFood GMO and Authentication Kit (Promega, Madison, WI, USA). The 16S rRNA gene was amplified and 
sequenced using the Illumina MiSeq technology and the Earth Microbiome Project protocol with some slight modifications. Briefly, region V4 of the 16S rRNA gene was PCR-amplified from each sample using composite forward and reverse primers designed with the Golay errorcorrecting code, and used to tag the PCR products (27). The sequence of the forward primer

was:

\section{AATGATACGGCGACCACCGAGATCTACACGCTXXXXXXXXXXXXTATGGTAATT}

GTGTGYCAGCMGCCGCGGTAA-3'. The italicized sequence is the $5^{\prime}$ Illumina adapter, the

$12 \mathrm{X}$ sequence is the Golay barcode, the bold sequence is the primer pad, the italicized and bold sequence is the primer linker, and the underlined sequence is the conserved bacterial primer 515F. The sequence of the reverse primer (806R) was: 5'CAAGCAGAAGACGGCATACGAGATAGTCAGCCAGCCGGACTACNVGGGTWTCTA

AT- $3^{\prime}$. The italicized sequence is the $3^{\prime}$ reverse complement sequence of the Illumina adapter, the bold sequence is the primer pad, the italicized and bold sequence is the primer linker, and the underlined sequence is the conserved bacterial primer 806R. PCR reactions included the Hot Master PCR mix (Quantabio, Beverly, MA, USA), $0.2 \mathrm{mM}$ of each primer, and 10-100 ng template. The reaction conditions were $3 \mathrm{~min}$ at $95{ }^{\circ} \mathrm{C}$, followed by 30 cycles of $45 \mathrm{~s}$ at $95{ }^{\circ} \mathrm{C}$, $60 \mathrm{~s}$ at $50{ }^{\circ} \mathrm{C}$, and $90 \mathrm{~s}$ at $72{ }^{\circ} \mathrm{C}$ on a BioRad thermocycler. PCR products were quantified with the Quant-iT PicoGreen dsDNA assay. Then, a master DNA pool was generated from the purified products in equimolar ratios and purified with Ampure magnetic purification beads (Agencourt, Brea, CA, USA). The pooled product was quantified using the Quant-iT PicoGreen dsDNA assay and then sequenced using an Illumina MiSeq sequencer (paired-end reads, $2 \times 250 \mathrm{bp}$ ) at the Genom'IC sequencing facility of Cornell University.

Then, the 16S rRNA sequences were analyzed using Quantitative Insights Into Microbial Ecology (QIIME2, Flagstaff, AZCA, USA) version 2019.7. Sequences were demultiplexed and quality-filtered using the Dada2 method (28) with QIIME2 default parameters to detect and 
correct Illumina amplicon sequence data, and a table of QIIME2 artifacts was generated. Then, the $\alpha$-diversity of bacterial communities was assessed by calculating the Shannon's diversity index, and $\beta$-diversity was used to analyze the dissimilarity among the group membership and structure. Both weighted and unweighted UniFrac distances were reported according to the principal coordinates analysis (PCoA). Group differences in $\alpha$ - and $\beta$-diversity indices were calculated using the Kruskal-Wallis test and permutational multivariate analysis of variance (PERMANOVA), respectively. For taxonomic analysis, features were assigned to operational taxonomic units with a $99 \%$ threshold of pairwise identity to the Greengenes reference database 13.8 (29). Differential taxon abundance among groups was tested with the ANCOM approach and the QUIIME2 software. The W-value generated by ANCOM is a count of the number of sub-hypotheses (Aitchison's log-ratio) that are significantly different across the tested groups for a given taxon. Correlations between gut microbiota composition (initial relative abundance of families) and clinical parameters were calculated with the Spearman's correlation test and the GraphPad Prism software (version 7.0). Finally, the baseline fecal microbial profiles, comprising 977 features, were used to build a random forest model to investigate whether the intestinal microbiota profile can predict response to exercise. The receiving operating characteristic (ROC) curve profile and the area under the ROC curve (AUC) were used as the main indicators of the model performance.

Biochemical assays. Blood samples were taken one week before the program start (baseline values) and then 2-4 days after the last exercise session for the trained group or after the last week of the study period for the control group. After overnight fasting, a cannula was inserted in the antecubital vein, and whole blood was collected in EDTA- and fluoride-containing vacutainers tubes. The plasma concentration of total cholesterol, high-density lipoprotein cholesterol (HDL-C), low-density lipoprotein cholesterol (LDL-C), triglycerides (TG), 
ultrasensitive reactive $\mathrm{C}$ protein (usCRP), glucose, insulin, and glycated hemoglobin (HbA1c) were immediately measured at an analysis center. The HOMA-IR index was calculated using the formula: HOMA-IR $=\left[\right.$ Fasting glucose $\left(\right.$ mmol.L $\left.L^{-1}\right) \times$ Fasting insulin $\left.\left(\mu \mathrm{U} \cdot \mathrm{mL}^{-1}\right)\right] / 22.5(30)$.

Physical activity and dietary assessments. All participants were requested not to modify their dietary and physical activity habits during the 12-week study period Their usual weekly level of physical activity was determined at baseline and after the 12 -week study period using the French version of the GPAQ (25).

At baseline and at week 12, each participant filled in a 5-day food-intake diary (3 weekdays and 2 weekend days) that was evaluated by a dietician using a nutrition analysis software (Nutrilog ${ }^{\circledR}$, Marans, France). A telephone helpline was proposed to participants who experienced problems in completing the 5-day food-intake diary.

\section{Training program.}

The training program included HIIT + RT three times per week, for 12 weeks (total = 36 sessions). Supervised sessions (approximately 45 minutes/each) were carried out at the Center of Resources, Expertise and Performance in Sports (CREPS), generally on Monday, Wednesday and Friday morning, to allow a sufficient recovery period. Each training session was supervised by an experienced certified physical activity instructor. Heart rates were continuously recorded to reach the expected intensities, based on the pre-screening $\mathrm{VO}_{2} \mathrm{max}$ values $\left(\mathrm{HR} / \mathrm{VO}_{2}\right.$ relationship). For ethical reasons, this program was also proposed to the control group at the study end.

\section{Combined high-intensity interval training and resistance training $(H I I T+R T)$. HIIT was} always performed before RT to normalize the concurrent training effects (31). The training 
program was based on the protocol described by Dupuit et al. (11). The HIIT protocol consisted of repeated cycles of sprinting/speeding for $8 \mathrm{~s}$ followed by slow pedaling (30-40 rpm) for $12 \mathrm{~s}$ on a WattBike pro Concept 2 (with a freewheel and a double air and magnetic braking system). Resistance was low to facilitate acceleration and limit bicycle-wheel inertia. Resistance was controlled to reach $\sim 85 \%$ of each participant's $\mathrm{HR}_{\max }$ during the 20 -min session. HR was continuously monitored (A300, Polar, Finland) to control the intensity. Overall, the mean intensity during HIIT sessions corresponded to $89 \pm 5 \%$ of $\mathrm{HR}_{\max }$. All participants could complete the 20-min exercise program at this intensity after three sessions.

Participants learnt the resistance exercise techniques in two training session before the study initiation, to become acquainted with the exercises. Ten repetitions of each exercise with a low load were performed, to adopt the correct body position and range of motion (considering the individual limitations). If a participant could not perform the movement due to a functional limitation or joint pain, another exercise targeting the same muscle group was proposed. In addition, for each exercise requiring loads, the one-repetition maximum (1 RM) indirect method was determined, as previously described (32). The RT program included two different training circuits, inspired by Dupuit et al. (11), with ten exercises performed in the following order: leg press, bench press, knee extension, cable row, dumbbell calf raise, elbow flexion, abdominal muscle, triceps exercises with upper pulley, plank, bum exercises (Circuit 1), and knees extension, pullover, leg press, side raise with dumbbells, dumbbell calf raise, triceps exercises with upper pulley, hip thrust, chin rowing, plank to upright row (Circuit 2). Participants performed a single-set circuit, with a load of 8-12RM. During the training program, the exact percentage of 1RM was not controlled, and for each exercise the set was performed until failure (33), with 60 to 90 s rest period between exercises. The workouts were individually supervised by the same certified personal trainer. When participants managed to correctly execute more than 12 repetitions (posture, speed, and range of motion), the load was 
adjusted to remain in the planned repetition zone. Participants alternated between circuits every 3 weeks to minimize boredom and to create some variation in the exercise choice.

\section{Statistical analyses.}

All statistical analyses were carried out with the STATISTICA version 12.00 software (StatSoft Inc., Tulsa, OK, USA). Data are presented as the mean \pm standard deviation (SD). The data normal distribution was tested using the Kolmogorov-Smirnov test, and the homogeneity of variance was tested with the F-test. Data were log-transformed, when appropriate, before analysis. Two-way analysis of variance (ANOVA) with repeated measures was used to determine group and time effects, and group $\times$ time interactions. When a significant effect was found, post-hoc multiple comparisons were performed using the Newman-Keuls test. The effect size and statistical power were reported when significant main or interaction effects were detected. The effect size was assessed using the partial eta-squared $\left(\eta^{2}\right)$ and ranked as follows: $\sim 0.01=$ small effect, $\sim 0.06=$ moderate effect, $\geq 0.14=$ large effect $(34)$. Baseline values and changes (delta value) between baseline and the study end [delta: (12 weeks - baseline / baseline) $\times 100$ ] were also compared between groups, using one-way ANOVA. Differences with a P-value $\leq 0.05$ were considered statistically significant.

\section{RESULTS}

Participants' Characteristics. At the beginning of the protocol, 33 postmenopausal women were eligible (Fig. 1). Then, two were excluded for cardiorespiratory abnormalities, and two withdrew for personal reasons before randomization. Moreover, 12 women did not complete the study ( $\mathrm{n}=6 /$ group). This was explained by the COVID-19-related closure of the training center for 8 women. In total, 17 women completed the study (HIIT + RT: $n=8$, CONT: $n=9$, 
Fig. 1). At baseline, mean age (CONT: $60.9 \pm 4.8$ years; HIIT + RT: $58.8 \pm 5.3$ years), total body weight (HIIT + RT: $78.8 \pm 12.5 \mathrm{~kg}$; CONT: $80.3 \pm 11.1 \mathrm{~kg})$ and total \%FM (HIIT + RT: attended $97.5 \%$ of training sessions and their compliance with the training program was $99 \%$ $\pm 1 \%$. No adverse event was reported during testing or training in both groups.

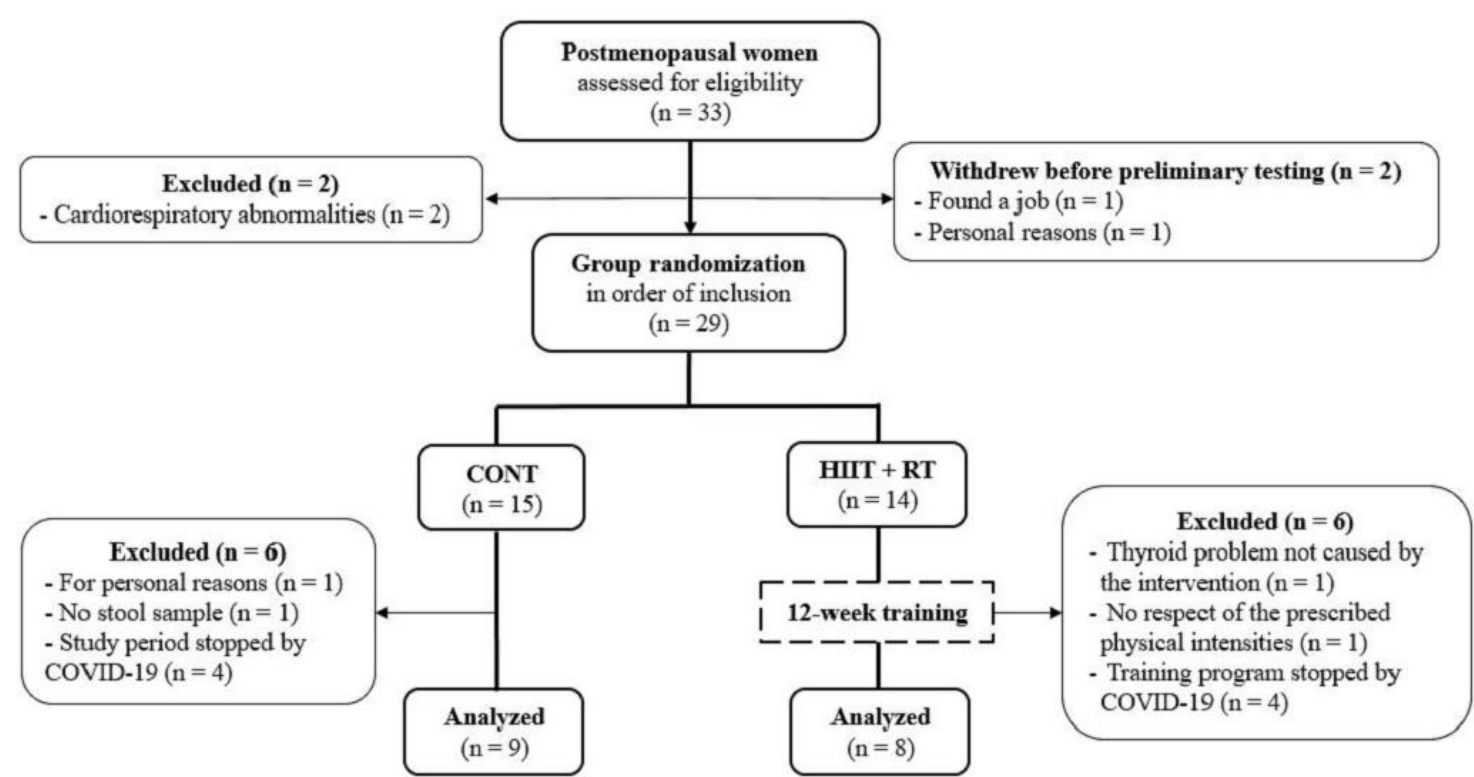

Figure 1: Study flowchart.

CONT: control group; HIIT + RT: high-intensity interval training + resistance training.

Habitual Energy Intake and Energy Expenditure. The pre- and post-training physical activity levels (GPAQ scores) were comparable between groups. The daily energy intake and the percentage of energy contribution from macronutrients did not significantly change during the intervention period in each group and was no different between groups (supplementary table 1).

Physical fitness. Baseline $\mathrm{VO}_{2 \max }\left(\mathrm{mL} \cdot \mathrm{kg}^{-1} \cdot \mathrm{min}^{-1}\right)$ and Peak Power Output (Watts $\cdot \mathrm{kg}^{-1}$ ) were not different between groups (Table 1). Overall, the baseline $\mathrm{VO}_{2 \max }$ value $\left(20.1 \pm 4.6 \mathrm{~mL} \cdot \mathrm{kg}^{-}\right.$ 
$\mathrm{VO}_{2 \max }$ and Peak Power Output (relative values) change were significantly higher in the HIIT

+ RT than CONT group (+ $14.5 \%$ vs. $+4 \%$ and $+17.8 \%$ vs. $-0.5 \%$, respectively; $\mathrm{p}<0.05)$

Anthropometric and body composition measurements. Baseline body mass and BMI did not differ between groups. Overall, the 12-week intervention induced an increase of these parameters (time effect, $\mathrm{p}=0.047, \eta^{2}=0.24 ; \mathrm{p}=0.048, \eta^{2}=0.24$, respectively). Total FM $(\mathrm{kg})$ and the percentage of total FM loss did not differ between groups at the study end (Fig. 2C). However, when expressed in percentage of body mass (\%BM), total FM was significantly decreased after the 12-week period (time effect, $p=0.045, \eta^{2}=0.24$, Table 1). Waist circumference (described as absolute value and percentage of change) only tended to decrease in the HIIT + RT group $\left(p=0.06\right.$ and $p=0.07$, respectively, with large size effects $\left(\eta^{2}=0.21\right.$ for both) (Table 1 and Fig. 2E). Overall, fat free mass and muscle mass (expressed as kg or $\% \mathrm{BW}$ ) were significantly increased (time effect, $\mathrm{p}<0.05$ ). However, the right thigh muscle mass was increased only in the HIIT + RT group $(+3.99 \% \pm 4.15)$ at the study end, leading to a significant difference with the CONT group $\left(p=0.047, \eta^{2}=0.24\right.$, Fig. 2D).

Abdominal and visceral fat mass. Baseline total abdominal ( $\mathrm{kg})$ and visceral FM (kg) were similar in the two groups. At the end of the training period, total abdominal FM was significantly reduced only in the HIIT + RT group $\left(p=0.007, \eta^{2}=0.39\right.$, Table 1$)$ and the percentage of abdominal FM change was significantly different between groups $\left(p=0.002, \eta^{2}\right.$ $=0.47$, Fig. $2 \mathrm{~F}$ ). The percentage of visceral FM change was negative only in the HIIT + RT group after the intervention $(-7.5 \% \pm 10.7)\left(p=0.035, \eta^{2}=0.26\right.$, Fig. $\left.2 G\right)$. Hence, the HIIT + RT protocol was sufficient to significantly and beneficially impact various anthropometric measurements. 

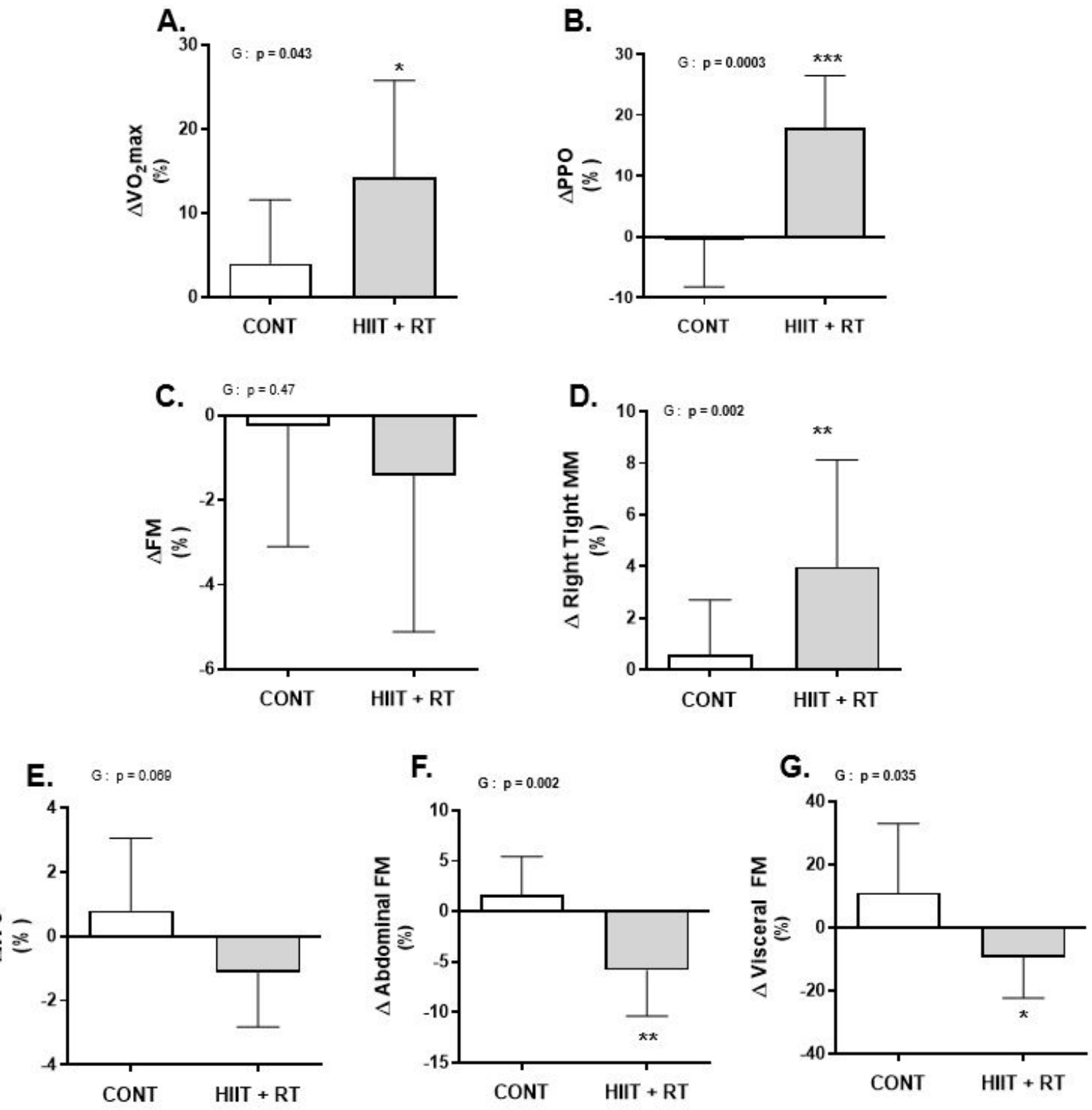

Figure 2: Changes of physical fitness (A-B), body composition (C-D), waist circumference 371 (E), abdominal (F) and visceral $(\mathrm{G})$ fat mass in the CONT $(n=9)$ and HIIT + RT $(n=8)$ groups between pre- and post-intervention. Data are the mean \pm SD.

373 CONT: control group; HIIT + RT: high-intensity interval training + resistance training; $\Delta$ : 374 change $=(12$ weeks - baseline $/$ baseline $) \times 100$; PPO: peak power output; FM: fat mass; MM: muscle mass; WC: waist circumference. $*: \mathrm{p} \leq 0.05, * *: \mathrm{p} \leq 0.005, * * *: \mathrm{p} \leq 0.0005:$ HIIT + RT vs. CONT group.

Metabolic profile. The blood parameters at baseline and at the end of the protocol are listed in Table 2. The 12-week intervention did not modify any of the tested metabolic parameters. 


\begin{tabular}{|c|c|c|c|c|c|c|c|}
\hline & \multicolumn{2}{|c|}{ CONT } & \multicolumn{2}{|c|}{ HIIT + RT } & \multicolumn{3}{|c|}{$\begin{array}{c}A N O V A(p) \\
\eta^{2}\end{array}$} \\
\hline & Pre & Post & Pre & Post & $G$ & $T$ & $G \times T$ \\
\hline \multicolumn{8}{|l|}{$\underline{\text { Body composition }}$} \\
\hline BMI $\left(\mathrm{kg} \cdot \mathrm{m}^{-2}\right)$ & $31.5 \pm 3.4$ & $31.7 \pm 3.3$ & $30.3 \pm 3.5$ & $30.6 \pm 3.5$ & $\begin{array}{l}0.30 \\
0.07\end{array}$ & $\begin{array}{c}0.048 \\
0.24\end{array}$ & $\begin{array}{c}0.80 \\
0.00\end{array}$ \\
\hline Body mass $(\mathrm{kg})$ & $80.3 \pm 11.2$ & $79.3 \pm 10.7$ & $77.8 \pm 12.4$ & $79.3 \pm 12.5$ & $\begin{array}{c}0.78 \\
0.01\end{array}$ & $\begin{array}{c}0.047 \\
0.24\end{array}$ & $\begin{array}{r}0.87 \\
0.00\end{array}$ \\
\hline Waist circumference $(\mathrm{cm})$ & $102.8 \pm 8.6$ & $103.1 \pm 10.2$ & $102.1 \pm 12.2$ & $101.0 \pm 12.3$ & $\begin{array}{l}0.75 \\
0.01\end{array}$ & $\begin{array}{c}0.82 \\
0.00\end{array}$ & $\begin{array}{c}0.063 \\
\mathbf{0 . 2 1}\end{array}$ \\
\hline Total FM (kg) & $27.5 \pm 7.6$ & $27.0 \pm 7.2$ & $28.0 \pm 7.4$ & $27.5 \pm 6.5$ & $\begin{array}{l}0.47 \\
0.03\end{array}$ & $\begin{array}{l}0.19 \\
0.11\end{array}$ & $\begin{array}{l}0.49 \\
0.04\end{array}$ \\
\hline Total FM (\%BM) & $33.0 \pm 4.6$ & $32.2 \pm 4.0$ & $35.2 \pm 4.9$ & $34.3 \pm 4.0$ & $\begin{array}{l}0.29 \\
0.07\end{array}$ & $\begin{array}{c}0.045 \\
0.24\end{array}$ & $\begin{array}{l}0.64 \\
0.02\end{array}$ \\
\hline Total FFM $(\mathrm{kg})$ & $44.3 \pm 3.9$ & $45.4 \pm 4.3$ & $50.8 \pm 6.5$ & $51.9 \pm 7.0$ & $\begin{array}{c}0.73 \\
0.01\end{array}$ & $\begin{array}{c}0.012 \\
0.35\end{array}$ & $\begin{array}{l}0.67 \\
0.01\end{array}$ \\
\hline Total FFM (\%BM) & $54.5 \pm 4.6$ & $55.2 \pm 4$ & $64.8 \pm 4.9$ & $65.7 \pm 4.0$ & $\begin{array}{l}0.29 \\
0.07\end{array}$ & $\begin{array}{c}0.046 \\
0.24\end{array}$ & $\begin{array}{c}0.54 \\
0.03\end{array}$ \\
\hline Muscle mass (kg) & $42.7 \pm 3.7$ & $43.7 \pm 4.0$ & $48.8 \pm 6.4$ & $49.9 \pm 6.8$ & $\begin{array}{l}0.75 \\
0.01\end{array}$ & $\begin{array}{c}0.013 \\
0.34\end{array}$ & $\begin{array}{l}0.68 \\
0.01\end{array}$ \\
\hline Muscle mass (\%BM) & $52.5 \pm 4.7$ & $53.2 \pm 4.0$ & $62.3 \pm 4.8$ & $63.2 \pm 3.9$ & $\begin{array}{l}0.33 \\
0.06\end{array}$ & $\begin{array}{c}0.039 \\
0.26\end{array}$ & $\begin{array}{l}0.52 \\
0.03\end{array}$ \\
\hline Arm muscle mass (kg) & $4.1 \pm 0.3$ & $4.0 \pm 0.3$ & $4.2 \pm 0.6$ & $4.4 \pm 0.8^{*}$ & $\begin{array}{l}0.34 \\
0.06\end{array}$ & $\begin{array}{l}0.59 \\
0.02\end{array}$ & $\begin{array}{l}0.05 \\
0.23\end{array}$ \\
\hline $\begin{array}{l}\text { Tight muscle mass } \\
\quad \text { (right side, } \mathrm{kg} \text { ) }\end{array}$ & $5.5 \pm 0.6$ & $5.5 \pm 0.6$ & $5.4 \pm 0.6$ & $5.6 \pm 0.6^{*}$ & 0.94 & 0.011 & 0.043 \\
\hline Total abdominal FM (kg) & $7.2 \pm 2.2$ & $7.4 \pm 2.2$ & $6.5 \pm 1.9$ & $6.0 \pm 1.7^{* * *}$ & $\begin{array}{c}0.00 \\
0.33 \\
0.06\end{array}$ & $\begin{array}{r}\mathbf{0 . 3 6} \\
0.13 \\
\mathbf{0 . 1 5}\end{array}$ & $\begin{array}{c}\mathbf{0 . 2 6} \\
\mathbf{0 . 0 0 7} \\
0.39\end{array}$ \\
\hline
\end{tabular}




$\begin{array}{llllllll}\text { Visceral FM }(\mathrm{kg}) & 4.0 \pm 1.5 & 4.3 \pm 1.5 & 3.2 \pm 0.6 & 2.8 \pm 0.5 & 0.07 & 0.53 & 0.07 \\ & & & & & \mathbf{0 . 2 0} & 0.03 & \mathbf{0 . 1 9}\end{array}$

\begin{tabular}{|c|c|c|c|c|c|c|c|}
\hline Physical fitness & & & & & & & \\
\hline $\mathbf{V O}_{2} \max \left(\mathrm{mL} \cdot \mathrm{kg}^{-1} \cdot \mathrm{min}^{-1}\right)$ & $19.7 \pm 3.6$ & $20.5 \pm 4.0$ & $20.5 \pm 5.7$ & $23.2 \pm 5.8 * *$ & $\begin{array}{l}0.46 \\
0.03\end{array}$ & $\begin{array}{c}0.001 \\
0.53\end{array}$ & $\begin{array}{c}0.037 \\
0.26\end{array}$ \\
\hline PPO (Watts) & $113 \pm 15$ & $114 \pm 21$ & $116 \pm 26$ & $136 \pm 23 * * *$ & $\begin{array}{c}0.24 \\
0.09\end{array}$ & $\begin{array}{c}\leq 10^{-3} \\
0.68\end{array}$ & $\begin{array}{c}\leq 10^{-3} \\
0.63\end{array}$ \\
\hline PPO (Watts $\cdot \mathrm{kg}^{-1}$ ) & $1.4 \pm 0.3$ & $1.4 \pm 0.3$ & $1.5 \pm 0.5$ & $1.8 \pm 0.5^{* * * *}$ & $\begin{array}{l}0.29 \\
0.07\end{array}$ & $\begin{array}{c}\leq 10^{-3} \\
0.67\end{array}$ & $\begin{array}{c}\leq 10^{-3} \\
0.68\end{array}$ \\
\hline
\end{tabular}

Table 1: Body composition and physical fitness in the CONT and HIIT + RT groups at baseline (pre) and at the end (post) of the 12-week intervention. Values are the mean \pm SD. G: group effect; T: time effect; $\mathrm{G} \times \mathrm{T}$ : group $\times$ time interaction.

BMI: body mass index; BM: body mass; FM: fat mass; FFM: free-fat mass; Muscle mass = FFM - Bone Mineral Content by dual-energy X-ray absorptiometry; PPO: peak power output.

$*: \mathrm{p} \leq 0.05, * *: \mathrm{p} \leq 0.005, * * * \mathrm{p} \leq 0.005$ (pre $v$ s. post in the same group) 


\begin{tabular}{|c|c|c|c|c|c|c|c|}
\hline & \multicolumn{2}{|c|}{ CONT } & \multicolumn{2}{|c|}{ HIIT + RT } & \multicolumn{3}{|c|}{ 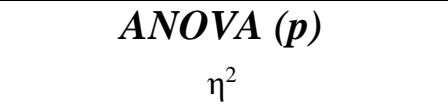 } \\
\hline & Pre & Post & Pre & Post & $G$ & $T$ & $G \times T$ \\
\hline Glycemia $\left(\mathrm{mmol} \cdot \mathrm{L}^{-1}\right)$ & $6.39 \pm 3.24$ & $6.06 \pm 3.26$ & $6.22 \pm 1.62$ & $6.44 \pm 1.94$ & $\begin{array}{c}0.93 \\
0.00\end{array}$ & $\begin{array}{c}0.86 \\
0.00\end{array}$ & $\begin{array}{l}0.34 \\
0.06\end{array}$ \\
\hline Insulinemia $\left(\mu \mathrm{U} \cdot \mathrm{L}^{-1}\right)$ & $13.24 \pm 6.36$ & $10.81 \pm 6.12$ & $10.24 \pm 2.73$ & $10.17 \pm 4.44$ & $\begin{array}{l}0.43 \\
0.06\end{array}$ & $\begin{array}{l}0.22 \\
\mathbf{0 . 1 4}\end{array}$ & $\begin{array}{c}0.44 \\
0.06\end{array}$ \\
\hline HbA1c (\%) & $6.20 \pm 1.64$ & $6.14 \pm 1.61$ & $6.20 \pm 0.73$ & $6.24 \pm 0.65$ & $\begin{array}{l}0.94 \\
0.01\end{array}$ & $\begin{array}{c}0.83 \\
0.00\end{array}$ & $\begin{array}{l}0.34 \\
0.06\end{array}$ \\
\hline HOMA-IR & $3.76 \pm 2.16$ & $2.20 \pm 1.99$ & $2.93 \pm 1.46$ & $2.99 \pm 1.28$ & $\begin{array}{c}0.99 \\
0.00\end{array}$ & $\begin{array}{l}0.10 \\
0.21\end{array}$ & $\begin{array}{l}0.22 \\
0.12\end{array}$ \\
\hline Total cholesterol $\left(\mathrm{mmol} \cdot \mathrm{L}^{-1}\right)$ & $5.91 \pm 0.88$ & $5.57 \pm 0.88$ & $5.97 \pm 1.16$ & $5.45 \pm 1.23$ & $\begin{array}{l}0.94 \\
0.00\end{array}$ & $\begin{array}{l}0.07 \\
\mathbf{0 . 2 0}\end{array}$ & $\begin{array}{c}0.71 \\
0.01\end{array}$ \\
\hline HDL-C $\left(\mathrm{mmol} \cdot \mathrm{L}^{-1}\right)$ & $1.58 \pm 0.17$ & $1.54 \pm 0.21$ & $1.49 \pm 0.27$ & $1.44 \pm 0.25$ & $\begin{array}{l}0.35 \\
0.06\end{array}$ & $\begin{array}{l}0.35 \\
0.06\end{array}$ & $\begin{array}{c}0.90 \\
0.00\end{array}$ \\
\hline LDL-C $\left(\mathrm{mmol} \cdot \mathrm{L}^{-1}\right)$ & $3.72 \pm 0.76$ & $3.52 \pm 0.85$ & $3.84 \pm 1.04$ & $3.48 \pm 1.02$ & $\begin{array}{l}0.91 \\
0.00\end{array}$ & $\begin{array}{l}0.10 \\
0.17\end{array}$ & $\begin{array}{l}0.62 \\
0.02\end{array}$ \\
\hline $\mathbf{T G}\left(\mathrm{mmol} \cdot \mathrm{L}^{-1}\right)$ & $1.36 \pm 0.46$ & $1.13 \pm 0.32$ & $1.39 \pm 0.51$ & $1.16 \pm 0.38$ & $\begin{array}{c}0.86 \\
0.00\end{array}$ & $\begin{array}{l}0.09 \\
\mathbf{0 . 1 7}\end{array}$ & $\begin{array}{r}0.99 \\
0.00\end{array}$ \\
\hline Total cholesterol/HDL-C & $3.77 \pm 0.42$ & $3.66 \pm 0.68$ & $4.38 \pm 1.04$ & $3.81 \pm 0.72$ & $\begin{array}{l}0.26 \\
0.08\end{array}$ & $\begin{array}{c}0.054 \\
\mathbf{0 . 2 2}\end{array}$ & $\begin{array}{l}0.18 \\
0.11\end{array}$ \\
\hline $\operatorname{usCRP}\left(\mathrm{mg} \cdot \mathrm{L}^{-1}\right)$ & $4.50 \pm 2.57$ & $4.48 \pm 3.12$ & $2.48 \pm 1.70$ & $2.35 \pm 1.43$ & $\begin{array}{c}0.14 \\
0.16\end{array}$ & $\begin{array}{l}0.66 \\
0.02\end{array}$ & $\begin{array}{l}0.68 \\
0.011\end{array}$ \\
\hline
\end{tabular}

Table 2: Metabolic parameters in the CONT and HIIT + RT groups at baseline (Pre) and after (Post) the intervention.

Values are the mean \pm SD. G: group effect; T: time effect; $\mathrm{G} \times \mathrm{T}$ : group $\times$ time interaction.

HDL: high density lipoproteins; LDL: low density lipoproteins; C: cholesterol; TG: triglycerides; usCRP: ultrasensitive C reactive protein. 
394 Analysis of the fecal microbiota composition by $16 \mathrm{~S}$ rRNA sequencing revealed that the 395 baseline $\alpha$-diversity (Shannon's diversity index) was not different between groups, and was not 396 changed at the study end (Fig. 3A). Before and after the intervention, Firmicutes and 397 Bacteroidetes were the most abundant phyla in both groups (91.5\%). The taxonomy analysis did not reveal any significant group difference at the phylum and family levels after the 12week program (Fig. 3B).

400 On the other hand, B-diversity analysis by PCoA of the unweighted Unifrac distance matrices 401 showed that the pre- and post-intervention microbiota composition changed in most patients 402 from the HIIT + RT group (Fig. 3C), whereas it remained stable in the CONT group (Fig. 3D).

403 This was confirmed by the bigger Bray-Curtis distance measured between pre- and post404 intervention in the HIIT + RT group (Fig. 3E), demonstrating the ability of the HIIT + RT protocol to modulate fecal microbiota.

A.

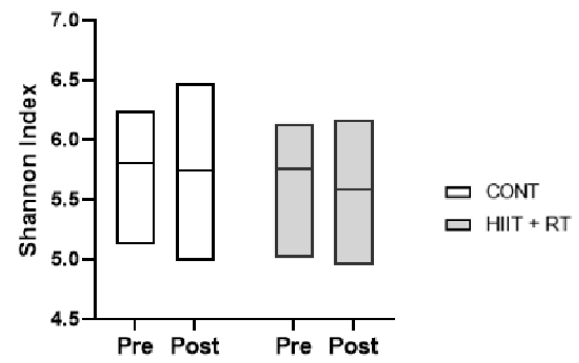

B.

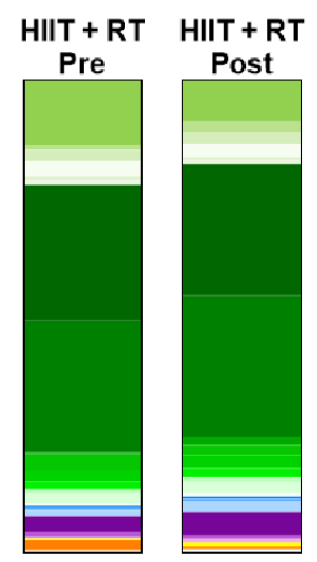

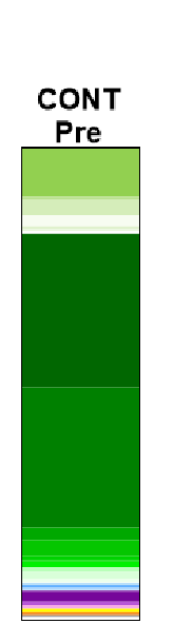

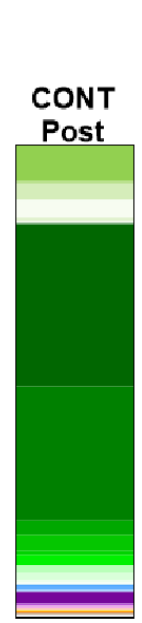

$\square$ Bacteroidaceae

$\square$ Paraprevotellacea

$\square$ Rikenellaceae

$\square$ Prevotellaceae

$\square$ Barnesiellaceae

$\square$ Unidentified Bacteroidetes

- Ruminococcaceae

Unidentified Clostridiale

$\square$ Erysipelotrichaceae

$\square$ Streptococcaceae

$\square$ Clostridiaceae

$\square$ Peptostreptococcaceae

$\square$ Veillonellaceae

$\square$ Christensenellaceae

$\square$ Others Firmicutes

口 Alcaligenaceae

$\square$ Desulfovibrionaceae

$\square$ Enterobacteriaceae

$\square$ Unidentified RF32

- Bifidobacteriaceae

$\square$ Coriobacteriaceae

$\square$ Verrucomicrobiaceae

$\square$ Undefined RF39

$\square$ Others

$\square$ Archea 

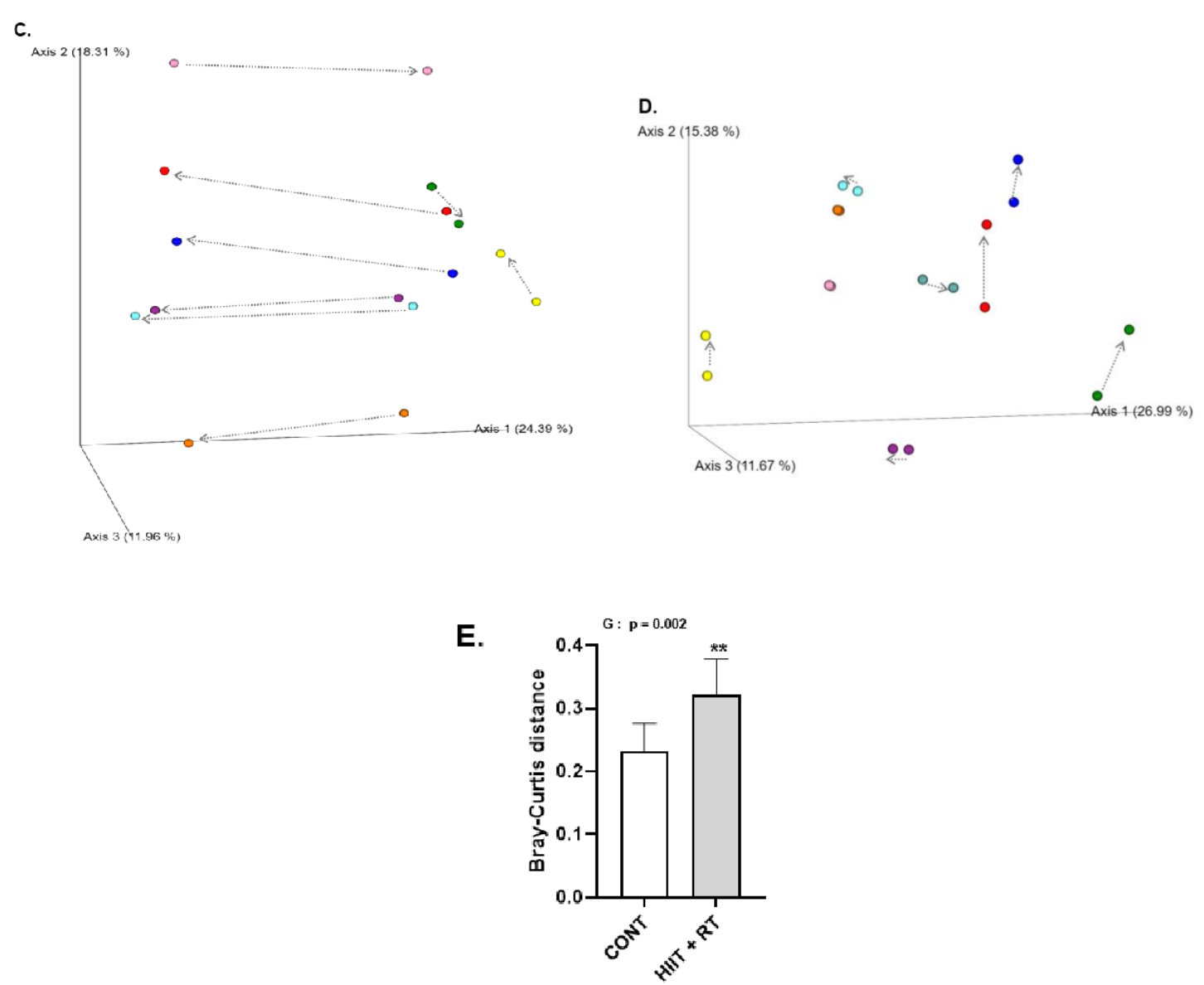

408 Figure 3: Changes of the $\alpha$-diversity and the $\beta$-diversity in the CONT $(n=9)$ and HIIT + RT 409 ( $n=8)$ groups between baseline (Pre) and study end (Post). (A) Shannon index, (B) Microbiota 410 composition with the relative abundance of each family, Principal Coordinates Analysis plots 411 of unweighted Unifrac distance metrics for the HIIT + RT (C) and CONT group (D), and (E) 412 Bray-Curtis distance between pre- and post-intervention in the HIIT + RT and CONT groups. 413 CONT: control group; HIIT + RT: high-intensity interval training + resistance training; $\rightarrow$ : pre414 to post-intervention.

As the PCoA plots (Fig. 3C-D) and the initial fecal microbiota composition (Supplementary Fig. 1) indicated a high interindividual variability in microbiota composition at baseline, then microbiota composition for each participant was normalized using their baseline composition to better highlight composition changes. This approach confirmed that $\alpha$-diversity variations were comparable between groups after the intervention (Fig. 4A), and revealed that overall,

421 fecal microbiota composition was changed in both groups at the study end (Fig. 4B). Moreover,

422 based on axis 2 and 3, the PCoA plots highlighted clear intervention-based clustering, 
424 (Fig. 4C).

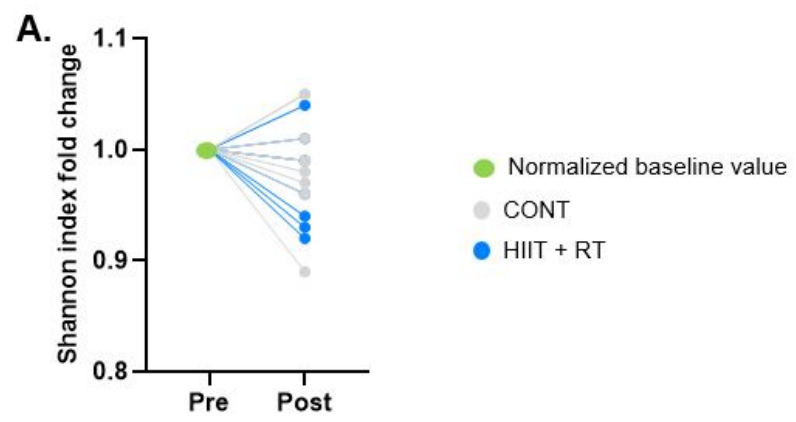

B.

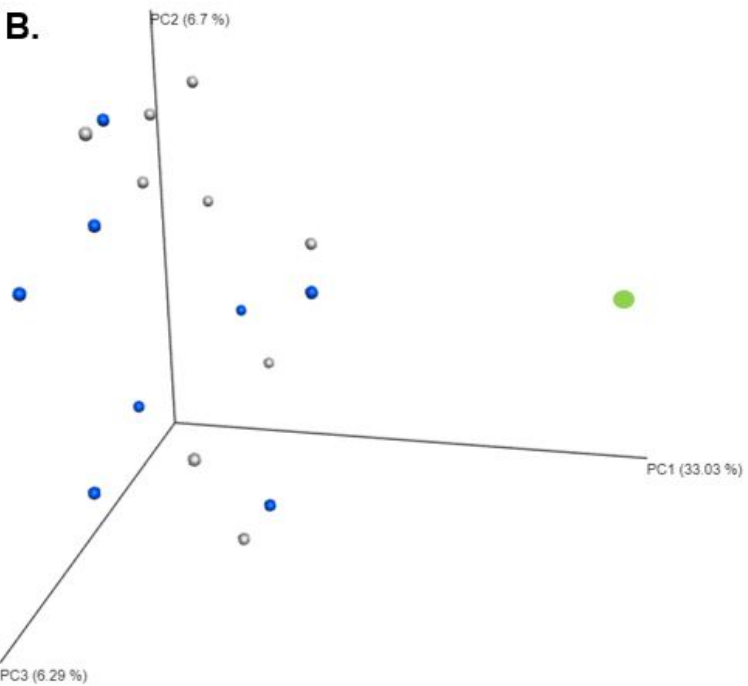

C.

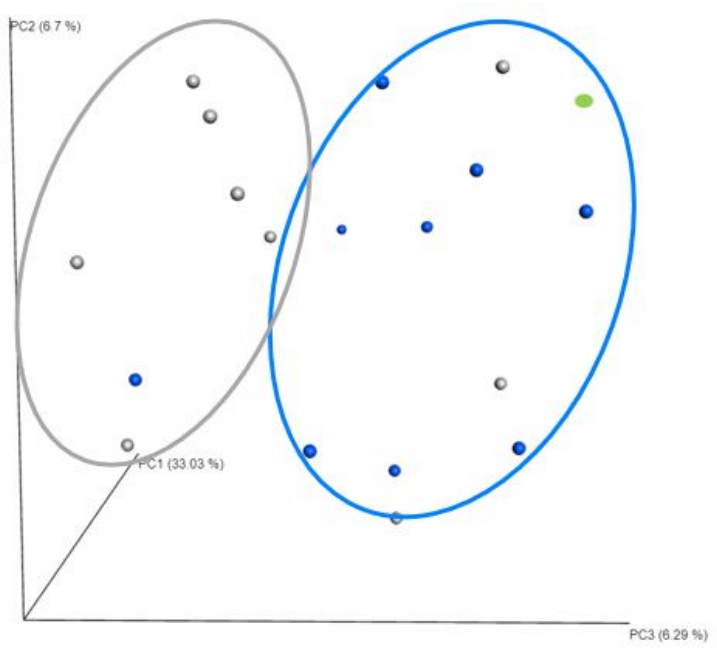

Figure 4: $\alpha-(\mathrm{A})$ and $\beta$-diversity (B-C) changes between baseline (Pre) and study end (Post) using the normalized baseline values for all CONT (gray points, $n=9$ ) and HIIT + RT (blue points, $n=8$ ) participants.

CONT: control group; HIIT + RT: high-intensity interval training + resistance training.

Next, a correlation analysis between the baseline relative abundance of specific microbiota families and changes in body composition and cardio-metabolic parameters in the HIIT + RT group (Fig. 5A-B) showed that Bifidobacteriaceae abundance was positively correlated with $\Delta \mathrm{FM}$ and negatively with $\Delta$ muscle mass and $\Delta \mathrm{HDL}-\mathrm{C}$. Conversely, Paraprevotellaceae and Prevotellaceae were negatively correlated with $\Delta \mathrm{FM}$ and positively with $\Delta$ muscle mass. These two families and Streptococcaceae and Desulfovibrionaceae were negatively correlated with changes in total cholesterol/HDL-C, unlike Lachnospiraceae and Ruminococcaccae. Alcaligenaceae were positively correlated with fasting glycemia, total cholesterol and LDL-C 
439 changes. Prevotellaceae was also positively correlated with PPO changes, and

440 Christensenellaceae relative abundance tended to be negatively correlated with changes in

441 WC. The Shannon's index was positively correlated with $\mathrm{VO}_{2 \max }$ changes (Fig. 5C), suggesting

442 an association between rich microbiota and cardiorespiratory fitness improvements.

443 As complementary observational data, to determine whether the training outcomes were related 444 to a specific baseline microbiota composition, HIIT + RT participants were grouped into 445 responders $(\mathrm{R}, n=3)$ and non-responders (NR, $n=5)$, based on the post-intervention total 446 abdominal FM loss (cut-off: -2.5\%; Fig. 6A). Then, a random forest algorithm that integrated 447 the baseline microbial features was used to investigate the ability of baseline microbiota 448 composition to predict the response to the HIIT + RT protocol. The obtained ROC curves (Fig. $4496 \mathrm{~B})$ had an AUC of 0.83 , indicating a relatively good ability of microbiota composition to 450 predict future HIIT + RT efficacy. Although the Shannon's diversity index did not show any 451 difference between R and NR when expressed as fold change (Fig. 6C), two clusters were 452 observed when using the $\beta$-diversity values that separated the R and NR participants, further suggesting that the baseline microbiota composition might predict the response to HIIT + RT (Fig. 6D-E). 


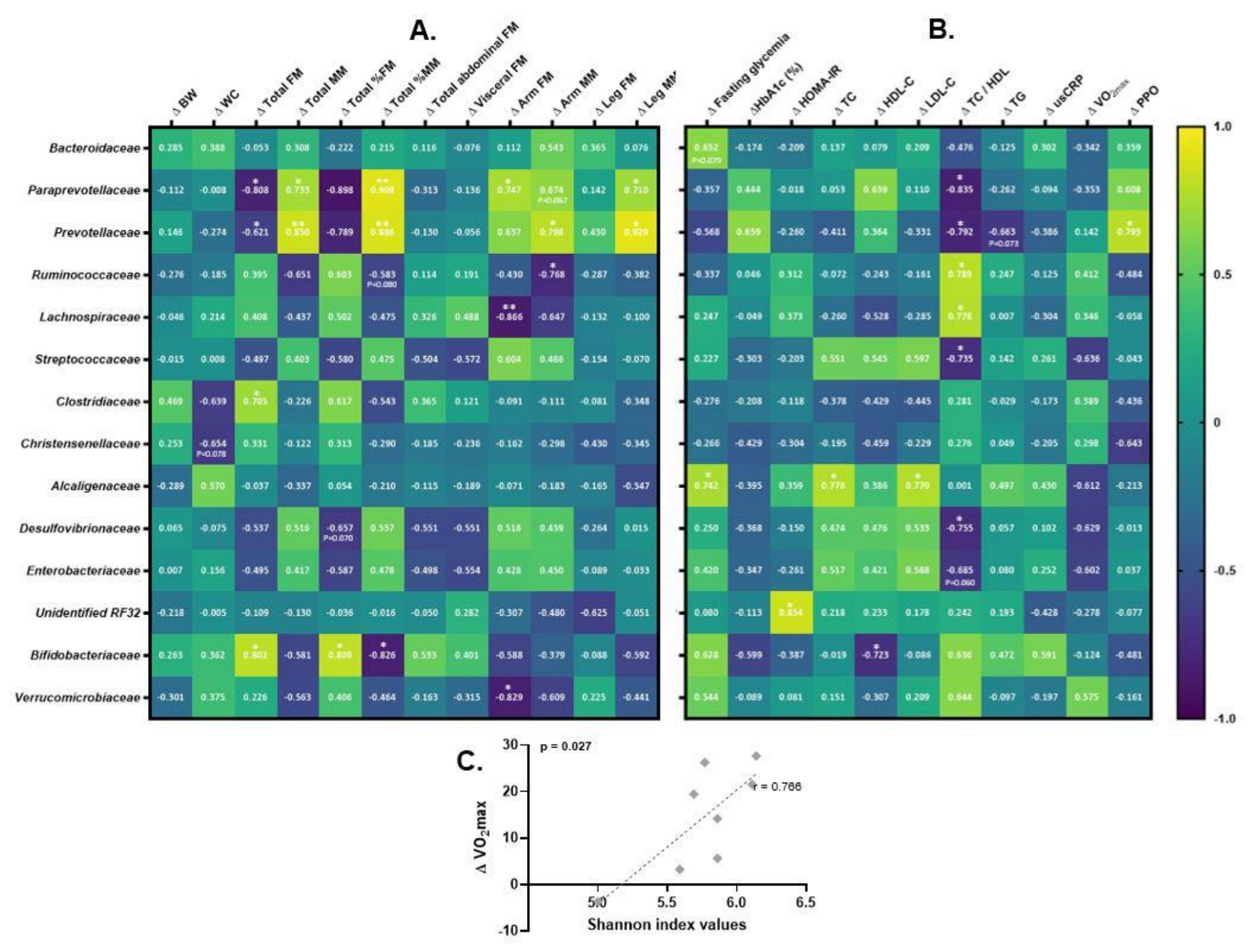


456 Figure 5: Associations between baseline relative abundance of specific microbiota families 457 and body composition (A) and cardio-metabolic parameters (B); and between $\alpha$-diversity and 458 physical fitness $(\mathrm{C})$ in the HIIT + RT group.

$459 \Delta$ : change between baseline and intervention end (\%), BW: body weight; FM: fat mass; MM: 460 muscle mass; WC: waist circumference; TC: total cholesterol; HDL: high density lipoproteins; 461 LDL: low density lipoproteins; C: cholesterol; TG: triglycerides; usCRP: ultrasensitive reactive 462 C protein; PPO: peak power output.

$463 * \mathrm{p}<0.05, * * \mathrm{p}<0.005, * * * \mathrm{p}<0.005$.

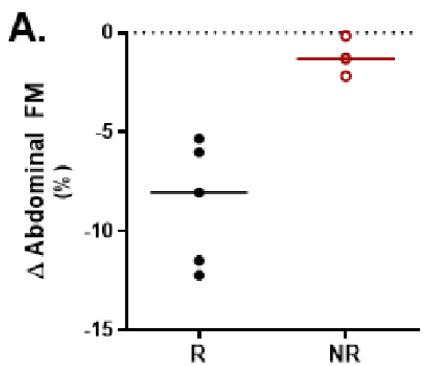

C.
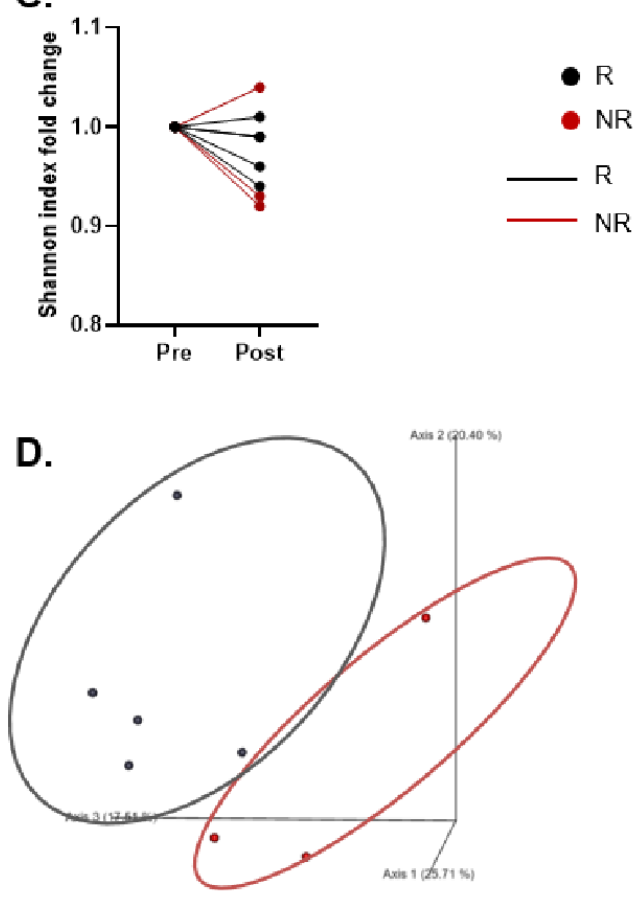

B.

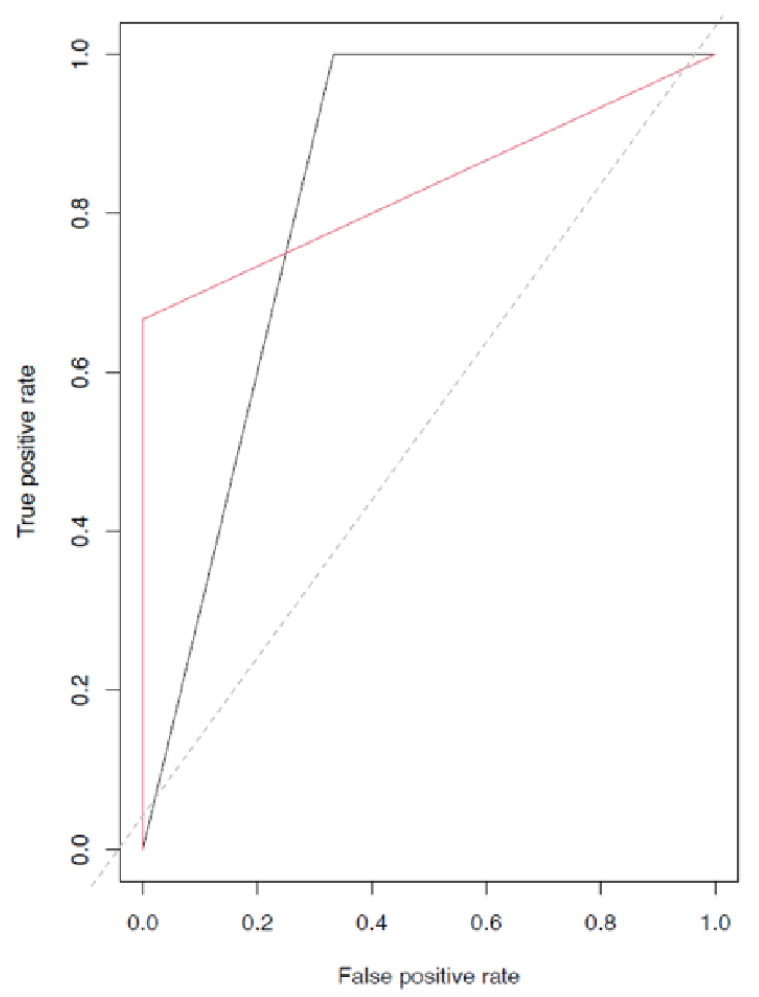




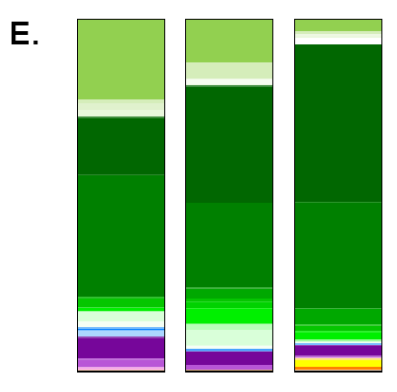

NR
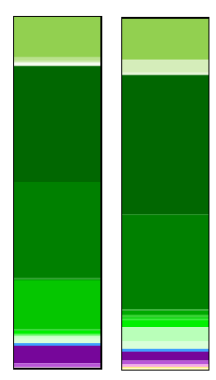

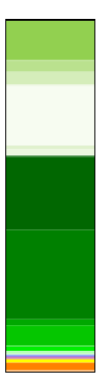

$\mathbf{R}$

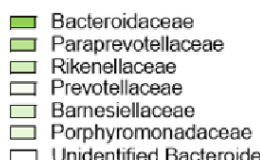

$\square$ Unidentified Bacteroidetes

- Ruminococcaceae

- Unidentified Clostridiales

Erysipelotrichaceae

Clostridiaccace

$\square$ Peptostreplocococaceae

$\square$ Christensenellacea

$\square$ Others Firmicutes

$\square$ Alcaligenaceae

$\square$ Desulfovibrionaceae

$\square$ Enterobacteriaceae

$\square$ Enterobacteriaceae

- Bifidobacteriaceae

$\square$ Coriobacteriaceae

$\square$ Verrucomicrobiaceae

$\square$ Undefined RF39

$\square$ Others

$\square$ Archea

467 Figure 6: Abdominal fat mass (FM) changes (\%) between baseline and study end in 468 participants from the HIIT + RT group divided in responders (R) and non-responders (NR) (A). 469 ROC curve representing the ability of microbiota composition to predict the response to 470 training in R and NR (B). $\alpha$ - and $\beta$-diversity analysis in R and NR (C-D). Individual basal microbiota composition at the family level in R and NR $(E)$.

\section{DISCUSSION}

body composition and fecal microbiota in non-dieting postmenopausal women with overweight or obesity. Compared with the control group, the HIIT + RT program significantly increased 479 physical fitness $\left(\mathrm{VO}_{2 \max }\right.$, Peak Power Output), decreased total abdominal and visceral FM, and enhanced segmental muscle mass. The fecal microbiota $\beta$-diversity significantly changed between pre- and post-intervention in the HIIT + RT group, and the Shannon's index was positively correlated with $\mathrm{VO}_{2 \max }$ changes. Notably, various intestinal microbiota components correlated with HIIT + RT-induced body composition changes, and baseline microbiota composition predicted the response to the HIIT + RT program. 
In women, menopause is associated with body composition modifications including FM

gain, particularly (intra-)abdominal adipose tissue, and muscle mass loss (35). FM accumulation and its adverse distribution (i.e. abdominal/visceral deposits) partly explain the higher CVD risks in this population $(7,8)$. Regular physical activity might be an efficient strategy to prevent and counteract estrogen deficiency-induced FM gain and abdominal FM deposit in postmenopausal women (35). However, the frequency of spontaneous physical activity seems to be lower in post- than in pre-menopausal women (6). In accordance with several reviews and meta-analyses $(36,37)$, our group demonstrated that HIIT is a safe and time-efficient strategy to reduce total and (intra-)abdominal FM in pre- and postmenopausal women, like in men $(38,39)$. Recently, our laboratory also showed that compared with HIIT alone, the HIIT + RT combination more effectively decreases (intra-)abdominal FM (kg and $\%)$ and increases total muscle mass (\%) in postmenopausal women with overweight/obesity (11). Using the same protocol $\left[60 \times 8\right.$ s at $80-90 \%$ of $H R_{\max }, 12$ s active recovery +10 wholebody resistance exercises: 1 set of 8-12 repetitions], we obtained similar results in the present study, indicating a large effect of HIIT + RT on total abdominal and visceral FM loss. Similarly, Rashti et al. observed a decrease in total abdominal FM (both visceral and subcutaneous adipose tissue assessed by MRI) after 10 weeks of HIIT $\left(4 \times\left(4^{\prime} 85-95 \% \mathrm{HR}_{\max } / 4^{\prime} 65 \% \mathrm{HR}_{\max }\right)\right.$ $+\mathrm{RT}\left(3-4 \times 8-15\right.$ repetitions $\mathrm{r}=30-60 \mathrm{~s}$ and $\left.\mathrm{R}=2-3^{\prime}\right)$ in active and non-active postmenopausal women (45-65 years) (12). However, HIIT and RT were not performed in the same session. Conversely, in older women (60-70 years), Yoon et al. did not observe any change in total and abdominal FM when the training included first RT $(9$ exercises $)$ and then HIIT $(10 \times 30$ s at 80 $90 \% \mathrm{HR}_{\text {reserve }} / 90 \mathrm{~s} 50-60 \% \mathrm{HR}_{\text {reserve }}$ ) in the same session, suggesting that the order of the training modalities may differently affect FM (40). A possible reason of these discrepancies is suggested in the review by Methenitis (31) showing that in concurrent training, the intra- 
session exercise sequence is important because the first pathway to be activated could inhibit the molecular adaptations induced by the second exercise mode. Concerning fat free mass, our study showed an increase of segmental muscle mass $(\mathrm{kg})$, corresponding to the trainingsolicited muscle groups. This muscle gain might enhance resting metabolic rate and therefore, the 24-h energy expenditure, favoring FM loss as part of the energy provided through lipid oxidation. As physical activity levels and total energy intakes remained unchanged during the study, our findings reinforces our conclusion that HIIT + RT on its own is an efficient strategy to modify body composition in postmenopausal women with overweight/obesity. The normal basal plasma metabolic values and HOMA-IR in both groups, despite overweight/obesity, might explain why the concurrent training failed to improve concomitantly the lipid profile and glucose homeostasis.

The fitness level of the postmenopausal women included in our study was evaluated using the $\mathrm{VO}_{2 \max }$ and PPO values. Lower cardiorespiratory fitness is associated with high BMI and increased CVD risks (41). Moreover, the increase in total and (intra-)abdominal adiposity that occurs after menopause is associated with lower $\mathrm{VO}_{2 \max }(42)$. Our training program significantly improved $\mathrm{VO}_{2 \max }$ and PPO. In the training group, $\mathrm{VO}_{2 \max }$ improvement was in the same range $(\sim 15 \%)$ as what was observed in postmenopausal women in the study by Dupuit et al. using exactly the same HIIT + RT protocol (11).

An increasing body of evidence suggests that gut microbiota can be rebalanced by exercise (15-17). Indeed, many studies reported that physical activity increases the number of beneficial microbial species, enriches microbiota diversity, and improves the development of commensal bacteria leading to health benefits (15). Lower microbiota diversity and higher Firmicutes/Bacteroidetes ratio have been associated with obesity, type 2 diabetes and impaired blood glucose (43). Our study was the first to examine the effect of a HIIT + RT program on microbiota composition in postmenopausal women. Our training intervention did not 
significantly change the $\alpha$-diversity and overall taxonomy of the fecal microbiota, but modulated the $\beta$-diversity. In humans, the absence of training effect on the $\alpha$-diversity is quite common, whereas the results on the $\beta$-diversity are more controversial (44). According to Shahar et al. 2020, physical activity-associated changes in $\beta$-diversity could be explained also by physical training-induced modifications of mitochondrial physiology and not only be the direct effects of exercise on the gastrointestinal tract. Mitochondrial physiology would not have the similar effect on $\alpha$-diversity (i.e. the microbiota community richness and evenness) (44). The mixed results concerning the effect of training on $\beta$-diversity (increase or not significant changes) may be partly ascribed also to the confounding effect of various factors, such as sex, age, diet, metabolic profile, fitness level and physical activity modality (duration, frequency and modes), that may also influence gut microbiota composition. Furthermore, the different collection and analysis techniques of the fecal microbiota may also bias comparisons (45). When studies using physical activity programs are compared, some authors did not find any $\beta$ diversity difference $(23,46-48)$, whereas others detected significant changes $(21,22)$. Our results support the ability of HIIT + RT to modulate fecal microbiota in a population of postmenopausal women with overweight or obesity. Similarly, Zhong et al. recently showed that a 2-month program $\left(4 \mathrm{~d} \cdot \mathrm{wk}^{-1}\right)$ of combined aerobic (steps: $4 \times 4$ ' $/ \mathrm{r}=20$ s, undefined intensity) and RT (elastic band: $2-3 \times 8-15$ repetitions) modifies the gut microbiota $\beta$-diversity in postmenopausal women (60-75 years) (49). Besides the classical richness and diversity, physical training may also modulate the relative abundance of specific phyla, families or bacterial species $(20,23,48)$. We did not detect such an effect. This could be due to the relatively small number of participants in our study. However, it is also worth noting that although the human gut microbiota is relatively stable at the phylum level, the microbial species and subspecies and their proportions may be specific to each person. In other words, human studies are characterized by a large heterogeneity of the gut/fecal microbiota (50). To overcome 
such difficulties, we normalized basal microbiota data in the two groups to better detect the potential effects of training. This strategy demonstrated that training may specifically modulate gut microbiota, as shown by the two distinct clusters in the PCoA plot. Moreover, to establish the association between baseline fecal microbiota composition and training-induced body composition and metabolic profile changes, we performed correlation analyses. Contrasting findings are observed in the literature regarding the effect of exercise interventions on fecal counts for the phyla which make comparisons difficult. Our analyses showed that Bifidobacteriaceae abundance was positively correlated with FM change. This result has been already shown by Munuka et al. in sedentary overweight women after six weeks of endurance training (21). Bifidobacteriaceae metabolize glucose and produce lactic acid and acetic acid (51) that regulates GPR41 and GPR43 (52), two short chain free fatty acid receptors with possibly a protective role against obesity. Conversely, Paraprevotellaceae and Prevotellaceae were negatively correlated with FM change (\%) and positively associated muscle mass change (\%). Interestingly, the Shannon's index was positively correlated with $\Delta \mathrm{VO}_{2 \max }$, suggesting an association between rich microbiota and cardiorespiratory fitness improvements. This confirms the findings by Estaki et al. showing a significant relationship between fecal microbiota diversity and $\mathrm{VO}_{2 \max }(53)$. On the other hand, Bycura et al. did not find any difference between pre-intervention microbiota composition and cardiorespiratory fitness changes after 8 weeks of cycling endurance training, free aerobic activities or resistance training (24).

Some people do not respond favorably to exercise. The term 'non-responder' is often used to describe the lack of response (to an exercise intervention) for a pre-specified outcome. As the main outcome of our study included (intra)-abdominal FM loss, we separated arbitrarily our training group in "responders" and "non-responders" based on the post-intervention total abdominal FM loss (-2.5\% cut-off). By using a random forest algorithm integrating baseline microbial features, our results showed that baseline microbiota composition can predict the 
response to the HIIT + RT protocol, thus strengthening the link between fecal microbiota composition and exercise-induced body composition changes.

One of the limitations of this study concerns the small number of participants. Indeed, our sample was sufficient to highlight, as expected, a significant (intra-)abdominal FM loss in the HIIT + RT group, but the high inter-individual variability in fecal microbiota composition made difficult the determination of the potential cross-talk between gut microbiota and adipose tissue after the training program (54). However, the normalization of the baseline fecal microbiota composition values and the analysis made in responders and non-responders strengthen our hypothesis. Another limitation was the lack of diet monitoring throughout the intervention period. Diet was recorded using a 5-day food-intake diary only at baseline and at week 12. We cannot guarantee that the diet did not fluctuate between these time points and/or that specific components were not included in the diet at some point, thus contributing to modulate gut microbiota composition (55). in postmenopausal women with overweight/obesity. We confirmed that a 12-week cycling HIIT + RT program decreases (intra-) abdominal FM, increases active muscle mass, and improves cardiorespiratory fitness in this population. These modifications were partly related to the exercise-induced modulation of the fecal microbiota. In addition, results of the current study also suggest that baseline gut microbiota composition might predict HIIT + RT efficiency. These findings must be confirmed in a larger sample, but could have potential implications for obesity management, both for physical activity professionals and also for nutritionists/dieticians who can modulate gut microbiota through the diet or specific dietary supplements. 
611 Acknowledgements

612 The authors want to thank all the study participants for their kind collaboration, Alban Bonnet 613 and Renaud Laurent for their kind assistance during the training sessions and their help in data 614 collection. We also thank Mélissa Kordahi for her help with statistical analysis.

\section{Conflict of interest}

617 The results of this study are presented clearly, honestly, and without fabrication, falsification, or inappropriate data manipulation. The results of the present study do not constitute endorsement by the American College of Sports Medicine.

620

621

\section{Competing interests}

622 The authors declare that they have no competing interests.

Authors' contributions

MD was the PhD student in the PACWOMan study and designed and supervised the different training modalities. She met all participants, carried out the anthropometric measurements, collected and analyzed all data, supervised training sessions, processed fecal samples, and wrote the first and subsequent drafts of the paper. MR was a co-investigator, and assisted with the study design. $\mathrm{CM}$ and $\mathrm{PB}$, physicians, assisted with the study design, and oversaw the medical aspects of the study. MD supervised training sessions and analyzed dietary. MD extracted DNA of fecal sample and BC analyzed microbiota by 16S rRNA gene sequencing using Illumina technology. NB conceived the study idea, was responsible for the overall study design, and for monitoring data collection. All authors read and approved the final manuscript. 
The PACWOMan Study was funded by the University of Clermont Auvergne (AME2P laboratory) and by the French government IDEX-ISITE initiative 16-IDEX-0001 (CAP 20-25), I-SITE project (CAP 2025) of the University of Clermont Auvergne. BC's laboratory is supported by a Starting Grant from the European Research Council (ERC) under the European Union's Horizon 2020 research and innovation program (grant agreement No. ERC-2018-StG804135), a Chaire d'Excellence from IdEx Université de Paris - ANR-18-IDEX-0001, and an design, the collection, analysis, and interpretation of data, the writing of the manuscript, and the decision to submit the article for publication.

\section{References}

1. De Lorenzo A., Van Bavel D., De Moraes R., Tibiriça E.V. High-intensity interval training or continuous training, combined or not with fasting, in obese or overweight women with cardiometabolic risk factors: Study protocol for a randomised clinical trial. BMJ Open [Internet]. 2018;8(4) doi:10.1136/bmjopen-2017-019304.

2. Haro C, Montes-Borrego M, Rangel-Zúñiga OA, et al. Two Healthy Diets Modulate Gut Microbial Community Improving Insulin Sensitivity in a Human Obese Population. J Clin Endocrinol Metab. 2016;101(1):233-42.

3. Lee HS, Lee J. Effects of Exercise Interventions on Weight, Body Mass Index, Lean Body Mass and Accumulated Visceral Fat in Overweight and Obese Individuals: A Systematic Review and Meta-Analysis of Randomized Controlled Trials. Int J Environ Res Public Health. 2021;18(5):2635.

4. Monteleone P, Mascagni G, Giannini A, Genazzani AR, Simoncini T. Symptoms of menopause global prevalence, physiology and implications. Nat Rev Endocrinol. 2018;14(4):199-215.

5. Abildgaard J, Pedersen AT, Green CJ, et al. Menopause is associated with decreased whole body fat oxidation during exercise. Am J Physiol Endocrinol Metab. 2013;304(11):E1227-1236.

6. Davis SR, Castelo-Branco C, Chedraui P, et al. Understanding weight gain at menopause. Climacteric. 2012;15(5):419-29.

7. Palmer BF, Clegg DJ. The sexual dimorphism of obesity. Molecular and Cellular Endocrinology. 2015;402:113-9. 
8. Manrique-Acevedo C, Chinnakotla B, Padilla J, Martinez-Lemus LA, Gozal D. Obesity and cardiovascular disease in women. Int J Obes (Lond). 2020;1210-26.

9. Álvarez C, Ramírez-Vélez R, Ramírez-Campillo R, et al. Improvements cardiometabolic risk factors in Latin American Amerindians (the Mapuche) with concurrent training. Scand J Med Sci Sports. 2019;29(6):886-96.

10. Maillard F, Rousset S, Pereira B, et al. High-intensity interval training reduces abdominal fat mass in postmenopausal women with type 2 diabetes. Diabetes Metab. 2016;42(6):433-41.

11. Dupuit M, Rance M, Morel C, et al. Moderate-Intensity Continuous Training or High-Intensity Interval Training with or without Resistance Training for Altering Body Composition in Postmenopausal Women. Med Sci Sports Exerc. 2020;52(3):736-45.

12. Rashti BA, Mehrabani J, Damirchi A, Babaei P. The influence of concurrent training intensity on serum irisin and abdominal fat in postmenopausal women. Prz Menopauzalny. 2019;18(3):16673.

13. Ballini A, Scacco S, Boccellino M, Santacroce L, Arrigoni R. Microbiota and Obesity: Where Are We Now? Biology (Basel) [Internet]. 2020;9(12) doi:10.3390/biology9120415.

14. Vrieze A, Holleman F, Zoetendal EG, de Vos WM, Hoekstra JBL, Nieuwdorp M. The environment within: how gut microbiota may influence metabolism and body composition. Diabetologia. 2010;53(4):606-13.

15. Campbell SC, Wisniewski PJ. Exercise is a Novel Promoter of Intestinal Health and Microbial Diversity. Exerc Sport Sci Rev. 2017;45(1):41-7.

16. Mailing LJ, Allen JM, Buford TW, Fields CJ, Woods JA. Exercise and the Gut Microbiome: A Review of the Evidence, Potential Mechanisms, and Implications for Human Health. Exerc Sport Sci Rev. 2019;47(2):75-85.

17. Mitchell CM, Davy BM, Hulver MW, Neilson AP, Bennett BJ, Davy KP. Does Exercise Alter Gut Microbial Composition? A Systematic Review. Med Sci Sports Exerc. 2019;51(1):160-7.

18. Ortiz-Alvarez L, Xu H, Martinez-Tellez B. Influence of Exercise on the Human Gut Microbiota of Healthy Adults: A Systematic Review. Clin Transl Gastroenterol. 2020;11(2):e00126.

19. Hampton-Marcell JT, Eshoo TW, Cook MD, Gilbert JA, Horswill CA, Poretsky R. Comparative Analysis of Gut Microbiota Following Changes in Training Volume Among Swimmers. Int J Sports Med. 2020;41(5):292-9.

20. Morita E, Yokoyama H, Imai D, et al. Aerobic Exercise Training with Brisk Walking Increases Intestinal Bacteroides in Healthy Elderly Women. Nutrients [Internet]. 2019;11(4) doi:10.3390/nu11040868.

21. Munukka E, Ahtiainen JP, Puigbó P, et al. Six-Week Endurance Exercise Alters Gut Metagenome That Is not Reflected in Systemic Metabolism in Over-weight Women. Front Microbiol. 2018;9:2323.

22. Kern T, Blond MB, Hansen TH, et al. Structured exercise alters the gut microbiota in humans with overweight and obesity-A randomized controlled trial. Int J Obes (Lond). 2020;44(1):12535 . 
23. Motiani KK, Collado MC, Eskelinen J-J, et al. Exercise Training Modulates Gut Microbiota Profile and Improves Endotoxemia. Med Sci Sports Exerc. 2020;52(1):94-104.

24. Bycura D, Santos AC, Shiffer A, et al. Impact of Different Exercise Modalities on the Human Gut Microbiome. Sports (Basel) [Internet]. 2021;9(2) doi:10.3390/sports9020014.

25. Hallal PC, Andersen LB, Bull FC, Guthold R, Haskell W, Ekelund U. Global physical activity levels: surveillance progress, pitfalls, and prospects. The Lancet. 2012;380(9838):247-57.

26. Skalsky AJ, Han JJ, Abresch RT, Shin CS, McDonald CM. Assessment of regional body composition with dual-energy X-ray absorptiometry in Duchenne muscular dystrophy: Correlation of regional lean mass and quantitative strength. Muscle \& Nerve. 2009;39(5):647-51.

27. Caporaso JG, Lauber CL, Walters WA, et al. Ultra-high-throughput microbial community analysis on the Illumina HiSeq and MiSeq platforms. The ISME Journal. 2012;6(8):1621-4.

28. Callahan BJ, McMurdie PJ, Rosen MJ, Han AW, Johnson AJA, Holmes SP. DADA2: Highresolution sample inference from Illumina amplicon data. Nat Methods. 2016;13(7):581-3.

29. McDonald D, Price MN, Goodrich J, et al. An improved Greengenes taxonomy with explicit ranks for ecological and evolutionary analyses of bacteria and archaea. ISME J. 2012;6(3):610-8.

30. Matthews DR, Hosker JP, Rudenski AS, Naylor BA, Treacher DF, Turner RC. Homeostasis model assessment: insulin resistance and $\beta$-cell function from fasting plasma glucose and insulin concentrations in man. Diabetologia. 1985;28(7):412-9.

31. Methenitis S. A Brief Review on Concurrent Training: From Laboratory to the Field. Sports (Basel) [Internet]. 2018;6(4) doi:10.3390/sports6040127.

32. Brzycki M. Strength Testing-Predicting a One-Rep Max from Reps-to-Fatigue. Journal of Physical Education, Recreation \& Dance. 2013.

33. Cadore EL, Pinto RS, Lhullier FLR, et al. Physiological effects of concurrent training in elderly men. Int J Sports Med. 2010;31(10):689-97.

34. Cohen J. A power primer. Psychol Bull. 1992;112(1):155-9.

35. Karvonen-Gutierrez C, Kim C. Association of Mid-Life Changes in Body Size, Body Composition and Obesity Status with the Menopausal Transition. Healthcare (Basel). 2016;4(3):42.

36. Türk Y, Theel W, Kasteleyn MJ, et al. High intensity training in obesity: a Meta-analysis. Obes Sci Pract. 2017;3(3):258-71.

37. Mattioni Maturana F, Martus P, Zipfel S, NIEß AM. Effectiveness of HIIE versus MICT in Improving Cardiometabolic Risk Factors in Health and Disease: A Meta-analysis. Med Sci Sports Exerc. 2021;53(3):559-73.

38. Dupuit M, Maillard F, Pereira B, Marquezi ML, Lancha AH, Boisseau N. Effect of high intensity interval training on body composition in women before and after menopause: a meta-analysis. Exp Physiol. 2020;105(9):1470-90.

39. Maillard F, Pereira B, Boisseau N. Effect of High-Intensity Interval Training on Total, Abdominal and Visceral Fat Mass: A Meta-Analysis. Sports Med. 2018;48(2):269-88. 
40. Yoon J-R, Ha G-C, Kang S-J, Ko K-J. Effects of 12-week resistance exercise and interval training on the skeletal muscle area, physical fitness, and mental health in old women. $J$ Exerc Rehabil. 2019;15(6):839-47.

41. Abate N. Obesity and cardiovascular disease: Pathogenetic role of the metabolic syndrome and therapeutic implications. Journal of Diabetes and its Complications. 2000;14(3):154-74.

42. Lynch NA, Ryan AS, Berman DM, Sorkin JD, Nicklas BJ. Comparison of VO2max and disease risk factors between perimenopausal and postmenopausal women. Menopause. 2002;9(6):45662.

43. Blandino G, Inturri R, Lazzara F, Di Rosa M, Malaguarnera L. Impact of gut microbiota on diabetes mellitus. Diabetes Metab. 2016;42(5):303-15.

44. Shahar RT, Koren O, Matarasso S, Shochat T, Magzal F, Agmon M. Attributes of Physical Activity and Gut Microbiome in Adults: A Systematic Review. Int J Sports Med. 2020;41(12):801-14.

45. Song E-J, Lee E-S, Nam Y-D. Progress of analytical tools and techniques for human gut microbiome research. J Microbiol. 2018;56(10):693-705.

46. Taniguchi $\mathrm{H}$, Tanisawa K, Sun X, et al. Effects of short-term endurance exercise on gut microbiota in elderly men. Physiol Rep. 2018;6(23):e13935.

47. Rettedal EA, Cree JME, Adams SE, et al. Short-term high-intensity interval training exercise does not affect gut bacterial community diversity or composition of lean and overweight men. Experimental Physiology. 2020;105(8):1268-79.

48. Liu Y, Wang Y, Ni Y, et al. Gut Microbiome Fermentation Determines the Efficacy of Exercise for Diabetes Prevention. Cell Metab. 2020;31(1):77-91.e5.

49. Zhong F, Wen X, Yang M, et al. Effect of an 8-week Exercise Training on Gut Microbiota in Physically Inactive Older Women. Int J Sports Med. 2021;42(7):610-23.

50. Lynch SV, Pedersen O. The Human Intestinal Microbiome in Health and Disease. N Engl J Med. 2016;375(24):2369-79.

51. Mattarelli P, Biavati B, Holzapfel WH, Wood BJ. The Bifidobacteria and Related Organisms: Biology, Taxonomy, Applications. Academic Press; 2017. 326 p.

52. Ozato N, Saito S, Yamaguchi T, et al. Blautia genus associated with visceral fat accumulation in adults 20-76 years of age. NPJ Biofilms Microbiomes. 2019;5:28.

53. Estaki M, Pither J, Baumeister P, et al. Cardiorespiratory fitness as a predictor of intestinal microbial diversity and distinct metagenomic functions. Microbiome. 2016;4(1):42.

54. Qian X-B, Chen T, Xu Y-P, et al. A guide to human microbiome research: study design, sample collection, and bioinformatics analysis. Chin Med J (Engl). 2020;133(15):1844-55.

55. Flint HJ, Scott KP, Louis P, Duncan SH. The role of the gut microbiota in nutrition and health. Nat Rev Gastroenterol Hepatol. 2012;9(10):577-89. 
782 Supplementary Figure 1: Individual basal microbiota composition at the family level in the 783 HIIT + RT $(n=8)(\mathrm{A})$ and CONT $(n=9)(\mathrm{B})$ groups.

784 CONT: control group; HIIT + RT: high-intensity interval training + resistance training.

785
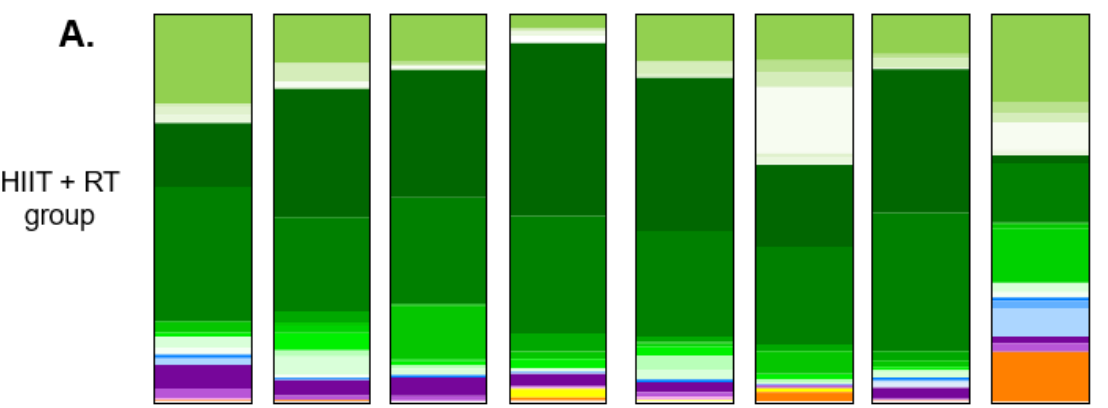

B.
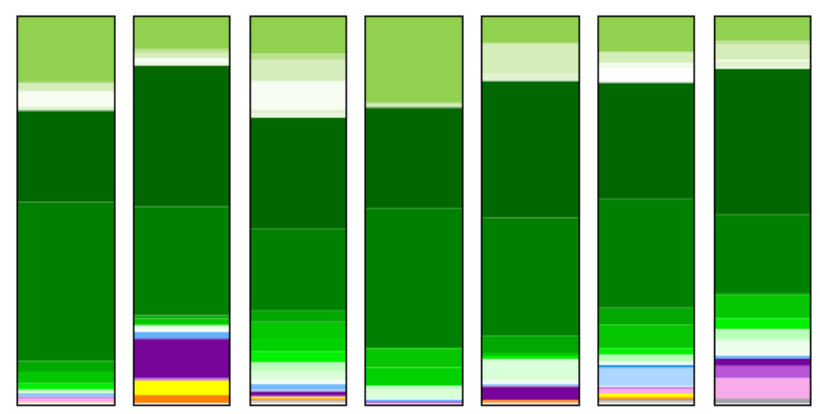

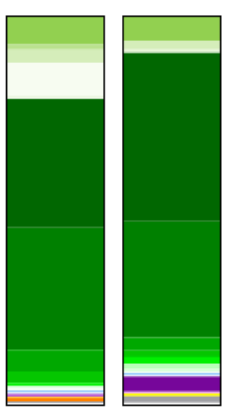


788 Supplementary Table 1: Mean daily energy intake and macronutrient repartition in the CONT 789 and HIIT + RT groups at baseline (pre) and at the end (post) of the 12-week intervention.

790 TEI: Total energy intake; CHO: carbohydrates; CONT: control group; HIIT + RT: high791 intensity interval training + resistance training.

792

\begin{tabular}{ccccc}
\hline & \multicolumn{2}{c}{ CONT } & \multicolumn{2}{c}{ HIIT + RT } \\
\cline { 2 - 5 } & Pre & Post & Pre & Post \\
\hline Energy intake (kcal) & $1608 \pm 287$ & $1682 \pm 366$ & $1551 \pm 239$ & $1447 \pm 251$ \\
CHO (\%TEI) & $37 \pm 7$ & $36 \pm 7$ & $43 \pm 7$ & $40 \pm 7$ \\
Fat (\%TEI) & $38 \pm 5$ & $40 \pm 7$ & $35 \pm 7$ & $38 \pm 4$ \\
Protein (\%TEI) & $20 \pm 5$ & $18 \pm 3$ & $18 \pm 4$ & $19 \pm 3$ \\
\hline
\end{tabular}

793

794

795 